\title{
Microscopic analysis of low-energy spin and orbital magnetic dipole excitations in deformed nuclei
}

\author{
V.O. Nesterenko ${ }^{1,2,3}$, P.I. Vishnevskiy ${ }^{1,2,4}$, J. Kvasil ${ }^{5}$, A. Repko ${ }^{6}$ and W. Kleinig ${ }^{1}$ \\ ${ }^{1}$ Laboratory of Theoretical Physics, Joint Institute for Nuclear Research, Dubna, Moscow region, 141980, Russid \\ 2 State University "Dubna", Dubna, Moscow Region, 141980, Russia \\ 3 Moscow Institute of Physics and Technology, Dolgoprudny, Moscow region, 141701, Russia \\ 4 Institute of Nuclear Physic, Almaty, 050032, Kazakhstan \\ 5 Institute of Particle and Nuclear Physics, Charles University, CZ-18000, Praha 8, Czech Republic and \\ 6 Institute of Physics, Slovak Academy of Sciences, 84511, Bratislava, Slovakia
}

(Dated: July 5, 2021)

\begin{abstract}
A low-energy magnetic dipole $(M 1)$ spin-scissors resonance (SSR) located just below the ordinary orbital scissors resonance (OSR) was recently predicted in deformed nuclei within the Wigner Function Moments (WFM) approach. We analyze this prediction using fully self-consistent Skyrme Quasiparticle Random Phase Approximation (QRPA) method. Skyrme forces SkM*, SVbas and SG2 are implemented to explore SSR and OSR in ${ }^{160,162,164}$ Dy and ${ }^{232} \mathrm{Th}$. Accuracy of the method is justified by a good description of M1 spin-flip giant resonance. The calculations show that isotopes ${ }^{160,162,164}$ Dy indeed have at $1.5-2.4 \mathrm{MeV}$ (below OSR) $I^{\pi} K=1^{+} 1$ states with a large $M 1$ spin strength ( $K$ is the projection of the total nuclear moment to the symmetry z-axis). These states are almost fully exhausted by $p p[411 \uparrow, 411 \downarrow]$ and $n n[521 \uparrow, 521 \downarrow]$ spin-flip configurations corresponding to $p p\left[2 d_{3 / 2}, 2 d_{5 / 2}\right]$ and $n n\left[2 f_{5 / 2}, 2 f_{7 / 2}\right]$ structures in the spherical limit. So the predicted SSR is actually reduced to low-orbital $(l=2,3)$ spin-flip states. Following our analysis and in contradiction with WFM spin-scissors picture, deformation is not the principle origin of the lowenergy spin $M 1$ states but only a factor affecting their features. The spin and orbital strengths are generally mixed and exhibit the interference: weak destructive in SSR range and strong constructive in OSR range. In ${ }^{232} \mathrm{Th}$, the $M 1$ spin strength is found very small. Two groups of $I^{\pi}=1^{+}$states observed experimentally at $2.4-4 \mathrm{MeV}$ in ${ }^{160,162,164} \mathrm{Dy}$ and at $2-4 \mathrm{MeV}$ in ${ }^{232} \mathrm{Th}$ are mainly explained by fragmentation of the orbital strength. Distributions of nuclear currents in QRPA states partly correspond to the isovector orbital-scissors flow but not to spin-scissors one.
\end{abstract}

PACS numbers: 13.40.-f, 21.60.Jz, 27.70.+q, 27.80.+w

\section{INTRODUCTION}

Magnetic dipole excitations in nuclei provide important information on the nuclear spin and orbital magnetism [1, 2]. For a long time, these excitations were mainly represented by $M 1(K=1)$ spin-flip giant resonance located at the energy $\mathrm{E} \approx 41 A^{-1 / 3} \mathrm{MeV}[1,2]$ and low-energy $M 1$ OSR with excitation energy $\mathrm{E} \approx$ $66 \delta A^{-1 / 3} \mathrm{MeV}$ 2] where $\delta$ is the parameter of nuclear axial quadrupole deformation. Both resonances are isovector and characterized by enhanced $M 1(\Delta K)$ transitions to the ground state.

The spin-flip resonance is produced by particle-hole spin-flip transitions between spin-orbit partners in the proton and neutron single-particle spectra. This resonance is related to spin nuclear magnetic properties and it exists in both spherical and deformed nuclei 1, 2]. The spin-flip resonance was widely applied to test a spin channel in various self-consistent approaches (Skyrme, Gogny and relativistic) [2 -8] and to check tensor forces [3, 4, 9] and spin-orbit interaction [3-5, 8] .

OSR is macroscopically treated as scissors-like out-ofphase oscillations of proton and neutron deformed sub-

*Electronic address: nester@theor.jinr.ru

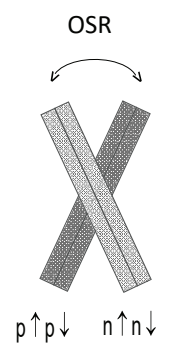

a)

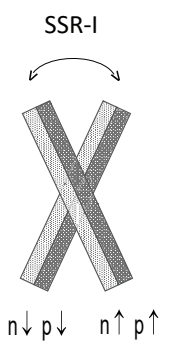

b)

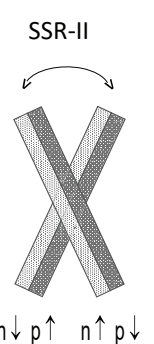

c)
FIG. 1: The schemes for the members of the scissors triple 28]: OSR (a), SSR-I (b) and SSR-II (c). The neutron (proton) axially deformed fractions are shown by light (dark) bars. The spin direction of nucleons is indicated by arrows. Each mode in the triple exhibits scissors-like oscillations of two blades: neutrons vs protons in OSR, spin-up vs spin-down nucleons in SSR-I (spins of neutrons and protons in each blade have the same direction), and SSR-II where neutron and proton spins in each blade have opposite directions.

systems, see Fig. 1a. This isovector resonance can exist only in deformed nuclei. It represents a remarkable example of a nuclear orbital magnetism. OSR was predicted in the two-rotor model [10, 11] and then experimentally observed in $\left(e, e^{\prime}\right)$ reaction [12]. OSR demonstrates some specific features: linear and square deformation laws for 


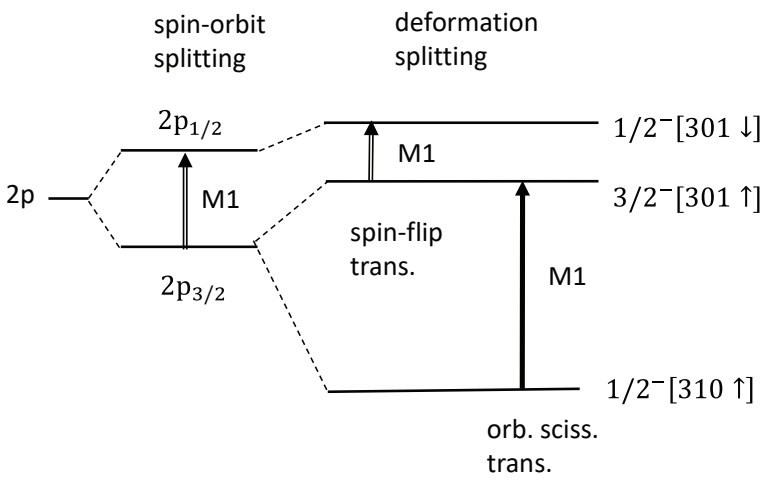

FIG. 2: A scheme of single-particle levels for $2 p$ subshell in spherical (left) and deformed (right) cases. The scheme corresponds to the proton $2 p$ subshell in ${ }^{162} \mathrm{Dy}$, calculated with the Skyrme force SG2. Spin-flip and orbital scissors $M 1$ transitions are exhibited by empty and filled arrows, respectively. In the deformed case, the levels are denoted by Nilsson asymptotic quantum numbers [37, 38], the arrows indicate spin direction.

its energy and strength, respectively [13, 14]. Various properties of OSR are outlined in reviews [2, 15, 16]. OSR is a kind of mixed-symmetry state [17 19]. Recent studies of OSR can be found elsewhere, see e.g. 20 22].

A decade ago, E.B. Balbutsev, I.V. Molodtsova, and P. Schuck have predicted (within the WFM method) that OSR should be supplemented by a low-energy spin scissor mode (SSR) 23]. Further WFM calculations with inclusion of the pairing 24,26 and isoscalar-isovector coupling in the residual interaction 27-29] have shown that SSR should have two branches, (see Fig. 1b,c) lying below OSR. Thus altogether the nuclear scissors mode should be a triplet: OSR + two SSR branches. All the scissors states should demonstrate significant $M 1(\Delta K=1)$ transitions to the ground state.

Following the WFM calculations, SSR should exist in medium and heavy axial deformed nuclei, typically at the excitation energy $E<2.7 \mathrm{MeV}$, i.e. just below OSR [25 29]. Many $I^{\pi}=1^{+}$states at $E<2.7 \mathrm{MeV}$ were already observed in rare-earth and actinide nuclei, see e.g. 3034 . However, they are usually not included in the experimental OSR systematics and their origin is still rather unclear. The prediction of SSR suggests an explanation for these states. Following the detailed WFM analysis for ${ }^{160,162,164} \mathrm{Dy},{ }^{232} \mathrm{Th}$ and ${ }^{236,238} \mathrm{U}[26-29]$, the nuclei ${ }^{164} \mathrm{Dy}$ and ${ }^{232} \mathrm{Th}$ are the most promising candidates for SSR. Low-energy $1^{+}$states in these nuclei form two distinctive groups which might be attributed to SSR and OSR.

The aim of the present paper is to scrutinize the WFM prediction of SSR from the microscopic viewpoint. It is well known that both orbital and spin-flip M1 transitions can be explained using single-particle schemes [1, 35]. An example of such scheme for $2 p$-subshell is shown in Fig. 2. This is a fraction of the proton scheme in ${ }^{162}$ Dy, calculated with Skyrme parametrization SG2 [36]. The computed equilibrium axial quadrupole deformation is $\beta_{2}=0.346$. The left part of the figure shows the splitting of $2 p$-subshell into $2 p_{1 / 2}$ and $2 p_{3 / 2}$ levels due to spin-orbit interaction. Already in this spherical case, a spin-flip $M 1$ transition between the levels is possible. The large deformation significantly splits the level $2 p_{3 / 2}$ and upshifts the level $2 p_{1 / 2}$ (right part of Fig. 2). In this case, two $M 1(\Delta K=1)$ transitions are possible: spin-flip $3 / 2^{-}[301 \uparrow] \rightarrow 1 / 2^{-}[301 \downarrow]$ and orbital $1 / 2^{-}[310 \uparrow] \rightarrow 3 / 2^{-}[301 \uparrow]$. The former connects the spin-orbit partners, the latter relates the levels arising due to deformation splitting. So we get two natural candidates for SSR and OSR. Because of the large deformation splitting, the orbital transition has a larger energy than the spin-flip one. So SSR should lie lower by energy than OSR.

As seen in Fig. 1(b,c), neutrons and protons in the left and right scissors blades have opposite spin directions. Perhaps, the predicted SSR can be somehow related to spin-flip excitations in neutron and proton spectra. This point is yet unclear (see discussion in Appendix B). What is important, Fig. 2 clearly shows that nuclear deformation is not the primary origin of low-energy spin-flip states (though it can significantly affect their features). This means that WFM interpretation of low-energy spin states in terms of deformation-induced scissors oscillations is questionable.

The main aim of the present study is to show that the predicted low-energy spin states are ordinary spinflip excitations and the available experimental data can be explained by the fragmentation of spin-flip and orbital M1 strength. Our analysis is performed for axially deformed nuclei ${ }^{160,162,164}$ Dy and ${ }^{232} \mathrm{Th}$. As mentioned above, two of these nuclei, ${ }^{164} \mathrm{Dy}$ and ${ }^{232} \mathrm{Th}$, are considered by WFM as promising candidates for SSR. The calculations are performed using fully self-consistent QRPA 39 44] with the Skyrme forces SG2 [36], SkM* [45], and SVbas [46]. As shown below, the spin and orbital lowenergy $M 1$ excitations are strongly mixed. So we will analyze both SSR and OSR. To demonstrate accuracy of our calculations, we will also present results for $M 1$ spin-flip giant resonance.

The paper is organized as follows. In Sec. II, the calculation scheme is outlined. In Sec. III, results of the calculations are discussed. In particular, flows the nuclear currents are exhibited. In Sec. IV, the conclusions are done. In Appendix A, a description of the $M 1$ spinflip giant resonance is illustrated. In Appendix B, some important aspects of WFM/QRPA comparison are commented. In Appendix C, expressions for the orbit and spin transition matrix elements are given.

\section{CALCULATION SCHEME}

The calculations are performed within the Skyrme QRPA model [39 44]. The model is fully self-consistent, 
TABLE I: Isoscalar effective mass $m_{0}^{*}$, isoscalar and isovector spin-orbit parameters $b_{4}$ and $b_{4}^{\prime}$, proton and neutron pairing constants $G_{p}$ and $G_{n}$, and the type of pairing in Skyrme forces $\mathrm{SkM}^{*}, \mathrm{SVbas}$, and SG2.

\begin{tabular}{|c|c|c|c|c|c|l|}
\hline force & $m_{0}^{*}$ & $\begin{array}{c}b_{4} \\
\mathrm{MeV} \mathrm{fm}^{5}\end{array}$ & $\begin{array}{c}b_{4}^{\prime} \\
\mathrm{MeV} \mathrm{fm}^{5}\end{array}$ & $\begin{array}{c}G_{p} \\
\mathrm{MeV} \mathrm{fm}^{3}\end{array}$ & $\begin{array}{c}G_{n} \\
\mathrm{MeV} \mathrm{fm}^{3}\end{array}$ & pairing \\
\hline SkM $^{*}$ & 0.79 & 65.0 & 65.0 & 279.08 & 258.96 & volume \\
\hline SVbas & 0.90 & 62.32 & 34.11 & 674.62 & 606.90 & surface \\
\hline SG2 & 0.79 & 52.5 & 52.5 & 296.76 & 259.58 & volume \\
\hline
\end{tabular}

TABLE II: Calculated parameters $\beta$ of the equilibrium axial quadrupole deformation vs the experimental values [49].

\begin{tabular}{|c|c|c|c|c|}
\hline Nucleus & \multicolumn{4}{|c|}{$\beta$} \\
\hline & SkM $^{*}$ & SVbas & SG2 & Exper. \\
\hline${ }^{160} \mathrm{Dy}$ & 0.339 & 0.331 & 0.339 & $0.334(2)$ \\
\hline${ }^{162} \mathrm{Dy}$ & 0.351 & 0.345 & 0.346 & $0.341(3)$ \\
\hline${ }^{164} \mathrm{Dy}$ & 0.354 & 0.348 & 0.352 & $0.349(3)$ \\
\hline${ }^{232} \mathrm{Th}$ & 0.256 & 0.247 & 0.238 & $0.248(6)$ \\
\hline
\end{tabular}

i.e.: i) both mean field and residual interaction are derived from the initial Skyrme functional, ii) the residual interaction takes into account all the terms of the Skyrme functional and Coulomb (direct and exchange) parts, iii) both particle-hole and particle-particle channels are included [42]. Spurious admixtures caused by violation of the rotational invariance are removed using the technique SEBRPA (spuriosity extracted before RPA) [44].

A representative set of Skyrme forces is used. We employ the standard force $\mathrm{SkM}^{*}$ [45], the recently developed force SVbas [46], and the force SG2 [36] which is often used in analysis of magnetic excitations, see e.g. [4, 5, 47]. As seen from Table凹 these forces have different isoscalar $b_{4}$ and isovector $b_{4}^{\prime}$ parameters of the spin-orbit terms in the Skyrme functionals (see definitions of the parameters in Refs. [4, 40]). In SkM* and SG2, the usual convention $b_{4}=b_{4}^{\prime}$ is used while in SVbas a separate tuning of $b_{4}$ and $b_{4}^{\prime}$ is done. All three Skyrme forces reproduce, though with different degrees of accuracy, a two-hump structure of M1 spin-flip giant resonance in deformed nuclei [4, 5]. As shown in Appendix A, SVbas and especially SG2 give a nice description of this resonance. So these two Skyrme forces can be considered as the most relevant for the present study.

The nuclear mean field and pairing are computed with the code SKYAX [48] using a two-dimensional grid in cylindrical coordinates. The calculation box extends up to three times the nuclear radii, the grid step is 0.4 fm. The axial quadrupole equilibrium deformation is obtained by minimization of the energy of the system. As seen from Table II] the obtained values of the deformation parameter $\beta$ are in a good agreement with the experimental data [49], especially for SVbas. All the forces reproduce a grow of the deformation from ${ }^{160} \mathrm{Dy}$ to ${ }^{164} \mathrm{Dy}$.

Pairing is described by the zero-range pairing interac-
TABLE III: Proton and neutron pairing gaps $\Delta_{p}$ and $\Delta_{n}$ and energy of $2_{1}^{+}$state of the ground-state rotational band, calculated in ${ }^{162}$ Dy and ${ }^{232}$ Th with Skyrme forces SkM*, SVbas, and SG2. The experimental data for the energy $E_{2_{1}^{+}}$are taken from database [49].

\begin{tabular}{|c|c|c|c|c|c|}
\hline Nucleus & & SkM $^{*}$ & SVbas & SG2 & exper. \\
\hline \multirow{3}{*}{${ }^{162}$ Dy } & $\Delta_{p}[\mathrm{MeV}]$ & 0.55 & 0.69 & 0.72 & \\
& $\Delta_{n}[\mathrm{MeV}]$ & 0.62 & 0.95 & 0.87 & \\
& $E_{2_{1}}[\mathrm{keV}]$ & 67.9 & 92.7 & 88.8 & 80.7 \\
\hline \multirow{3}{*}{${ }^{232} \mathrm{Th}$} & $\Delta_{p}[\mathrm{MeV}]$ & 0.53 & 0.61 & 0.75 & \\
& $\Delta_{n}[\mathrm{MeV}]$ & 0.54 & 0.80 & 0.78 & \\
& $E_{2_{1}^{+}}[\mathrm{keV}]$ & 41.2 & 57.1 & 63.0 & 49.4 \\
\hline
\end{tabular}

tion [51]

$$
V_{\text {pair }}^{q}\left(\mathbf{r}, \mathbf{r}^{\prime}\right)=G_{q}\left[1-\eta\left(\frac{\rho(\mathbf{r})}{\rho_{\text {pair }}}\right)\right] \delta\left(\mathbf{r}-\mathbf{r}^{\prime}\right)
$$

where $G_{q}$ are proton $(q=p)$ and neutron $(q=n)$ pairing strength constants. They are fitted to reproduce empirical pairing gaps obtained by the five-point formula along selected isotopic and isotonic chains [50]. The values of $G_{q}$ are shown in Table \. Further, $\rho(\mathbf{r})=\rho_{p}(\mathbf{r})+\rho_{n}(\mathbf{r})$ is the sum of proton and neutron densities. We get so-called volume pairing for $\eta=0$ and density-dependent surface pairing for $\eta=1$. As indicated in Table @ the former is used in $\mathrm{SkM}^{*}$ and $\mathrm{SG} 2$, and the latter is exploited in SVbas. In the latter case, we use SVbas parameter $\rho_{\text {pair }}=0.2011 \mathrm{fm}^{-3}$. Pairing correlations are included at the level of the iterative HF-BCS (Hartree-Fock plus Bardeen-Cooper-Schrieffer) method [42]. To cope with the divergent character of zero-range pairing forces, energy-dependent cut-off factors are used [42, 51].

Table III shows the calculated averaged proton and neutron pairing gaps $\Delta_{p}$ and $\Delta_{n}$ (defined in Eq. (30) of Ref. [51]) in ${ }^{162}$ Dy and ${ }^{232} \mathrm{Th}$. Also we exhibit the energies $E_{2_{1}^{+}}=3 \hbar^{2} / \mathcal{J}$ (with $\mathcal{J}$ being the nuclear moment of inertia) of $I^{\pi}=2^{+}$state in ground-state rotational band. These energies are sensitive to both deformation and pairing. As seen from Table III, SkM* underestimates while SVbas and SG2 somewhat overestimate the experimental $E_{2_{1}^{+}}$-values.

In our calculations, QRPA is implemented in the matrix form. A large configuration space is used. The singleparticle spectrum extends from the bottom of the potential well up to $30 \mathrm{MeV}$. For example, in SG2 calculations for ${ }^{162}$ Dy, 691 proton and 800 neutron single-particle levels are used. The two-quasiparticle (2qp) basis in QRPA calculation for $K^{\pi}=1^{+}$states includes 5270 proton and 9527 neutron configurations. We do not consider $K^{\pi}=0^{+}$excitations since it is well known [1, 2, 15, 16. that $M 1$ spin-flip and orbital-scissors modes are characterized by strong $M 1(\Delta K=1)$ transitions to the ground state.

Reduced probability for $M 1$ transitions from the ground state $|0\rangle$ with $I^{\pi} K=0^{+} 0$ to the excited QRPA 
state $|\nu\rangle$ with $I^{\pi} K=1^{+} 1$ reads

$$
B_{\nu}(M 1)=2|\langle\nu|\hat{\Gamma}(M 11)| 0\rangle|^{2} .
$$

The coefficient 2 means that contributions of both projections $K=1$ and -1 are taken into account. The transition operator has the form

$$
\hat{\Gamma}(M 11)=\mu_{N} \sqrt{\frac{3}{4 \pi}} \sum_{q=p, n}\left[g_{s}^{q} \hat{s}(\mu=1)+g_{l}^{q} \hat{l}(\mu=1)\right]
$$

where $\mu_{N}$ is the nuclear magneton, $\hat{s}(\mu=1)$ and $\hat{l}(\mu=1)$ are $\mu=1$ projections of the standard spin and orbital operators, $g_{s}^{q}$ and $g_{l}^{q}$ are spin and orbital gyromagnetic factors. We use the quenched spin g-factors $g_{s}^{q}=\eta \bar{g}_{s}^{q}$ where $\bar{g}_{s}^{p}=5.58$ and $\bar{g}_{s}^{n}=-3.82$ are bare proton and neutron g-factors and $\eta=0.7$ is the quenching parameter [1]. The orbital g-factors are $g_{l}^{p}=1$ and $g_{l}^{n}=0$. In what follows, we consider three cases: $\operatorname{spin}\left(g_{l}^{q}=0\right)$, orbital $\left(g_{s}^{q}=0\right)$, and total (when both spin and orbital transitions are taken into account). The expressions for orbital and spin M1 matrix elements are given in the Appendix C.

In deformed nuclei, electric and magnetic states with the same $K^{\pi}$ are mixed [1, 15, 35, 52]. In our case of $K^{\pi}=1^{+}$states, the magnetic dipole $M 1(K=1)$ and electric quadrupole $E 2(K=1)$ modes can be mixed. To estimate this mixing, we calculate reduced probability of E2 transitions $0^{+} 0 \rightarrow 2^{+} 1$ :

$$
B_{\nu}(E 2)=2|\langle\nu|\hat{\Gamma}(E 21)| 0\rangle|^{2}
$$

with the proton transition operator

$$
\hat{\Gamma}(E 21)=e r^{2} Y_{21}(\theta, \phi)
$$

where $Y_{21}(\theta, \phi)$ is the spherical harmonic.

We also calculate the current transition densities (CTD)

$$
\delta \mathbf{j}_{\nu}(\mathbf{r})=\langle\nu|\hat{\mathbf{j}}| 0\rangle(\mathbf{r})
$$

for the convective nuclear current

$$
\hat{\mathbf{j}}(\mathbf{r})=-i \frac{e \hbar}{2 m} \sum_{q=n, p} e_{\mathrm{eff}}^{q} \sum_{k \in q}\left(\delta\left(\mathbf{r}-\mathbf{r}_{k}\right) \nabla_{k}+\nabla_{k} \delta\left(\mathbf{r}-\mathbf{r}_{k}\right)\right) .
$$

Here $e_{\text {eff }}^{q}$ are the effective charges. They are $e_{\text {eff }}^{p}=1$ and $e_{\mathrm{eff}}^{n}=0$ for the proton current, $e_{\mathrm{eff}}^{p}=0$ and $e_{\mathrm{eff}}^{n}=1$ for the neutron current, $e_{\mathrm{eff}}^{p}=e_{\mathrm{eff}}^{n}=1$ for isoscalar current and $e_{\mathrm{eff}}^{p}=-e_{\mathrm{eff}}^{n}=1$ for isovector current.

Beside, we calculate the separate spin-up and spindown parts of CTD (6). For this aim, the wave function of the QRPA state $|\nu\rangle$ is projected to the proper spin direction using spinor structure of the involved singleparticle wave functions in cylindric coordinates, see Eqs. (C1)- C2 in Appendix C.

\section{RESULTS AND DISCUSSION}

\section{A. $\quad$ M1 strength in ${ }^{160,162,164}$ Dy}

In Figure 3, we compare calculated orbital, spin and total M1 strengths (2) in ${ }^{160,162,164}$ Dy with experimental data from the nuclear resonance fluorescence (NRF) reaction, see Refs. [30] for ${ }^{160} \mathrm{Dy}$ and [31] for ${ }^{162,164} \mathrm{Dy}$. QRPA results are obtained for the force SG2. Following the discussion in Sec. II and results for the spin-flip $M 1$ giant resonance in Appendix A, this force seems to be the most relevant for our analysis.

The plots (a-c) of the figure show that $M 1$ strength above $2.4 \mathrm{MeV}$ is mainly orbital. This strength constitutes the OSR. Instead, a few states at $\mathrm{E}<2.4 \mathrm{MeV}$ exhibit a noticeable spin strength, see plots (d-f). Following prediction [25 29], these states are candidates for SSR. Comparing spin and orbital strengths with the total one (plots (g-i)), we see that spin and orbital modes have a strong interference, both destructive and constructive. These results take place for all three Dy isotopes.

Figure 3 shows that NRF data [30, 31] do not give $I^{\pi}=1^{+}$states at $E<2.39 \mathrm{MeV}$. As discussed in Refs. 33], this may be caused by troubles of traditional NRF experiments to separate transitions in this energy range from a sizable background. The early data for ${ }^{160} \mathrm{Dy}[30$ give $1^{+}$states only for $E>2.8 \mathrm{MeV}$, though the level list in database [49] suggests many candidates for $1^{+}$states at lower excitation energies.

In ${ }^{162,164}$ Dy, NRF data 31] give two groups of $1^{+}$ states located above and below $2.7 \mathrm{MeV}$. The former group is usually treated as OSR. The latter is treated by WFM as SSR 25-29]. Note that low-energy groups of $1^{+}$ states were earlier observed in various rare-earth nuclei 30]. Recent Oslo $(\gamma, n)$ experiments [33] show that, in ${ }^{164}$ Dy, $40-60 \%$ of $M 1$ strength at energy range $0-4 \mathrm{MeV}$ is located below $2.7 \mathrm{MeV}$. Moreover, in this nucleus the total measured $M 1$ strength at $0-4 \mathrm{MeV}$ achieves 6.17 $\mu_{N}^{2}$ 31] which substantially exceeds the values $3-4 \mu_{N}^{2}$ typical for OSR in well-deformed rare-earth nuclei. This observation was treated by WFM as a clear signature of SSR in ${ }^{164}$ Dy 25 29]. However, following our results in Fig. 3, the states at $2.4-2.7 \mathrm{MeV}$ give mainly orbital $M 11$ transitions and so should also belong to OSR. They are omitted in OSR systematics with the lower boundary 2.7 $\mathrm{MeV}$ 52 but taken into account for the lower boundary $2.5 \mathrm{MeV}$ [20]. So, by our opinion, the data of Oslo group cannot be considered as the argument in favor of SSR.

In Figure 4, we demonstrate the distribution of M1 strength in ${ }^{162} \mathrm{Dy}$, calculated with the forces $\mathrm{SkM}^{*}$, SVbas, and SG2. It is seen that, despite some deviations in details, all these three forces give qualitatively similar results. In all cases, there is the range $0-2.4 \mathrm{MeV}$ with an essential spin strength and the range 2.4-4.0 MeV with a dominant orbital strength. Fig. 4 also demonstrates E2 strength (4) for the same $K^{\pi}=1^{+}$states. This strength is large at $2.6-4.0 \mathrm{MeV}$ and negligible at $0-2.6 \mathrm{MeV}$. The former result is typical for OSR [15, 52]. This means that 

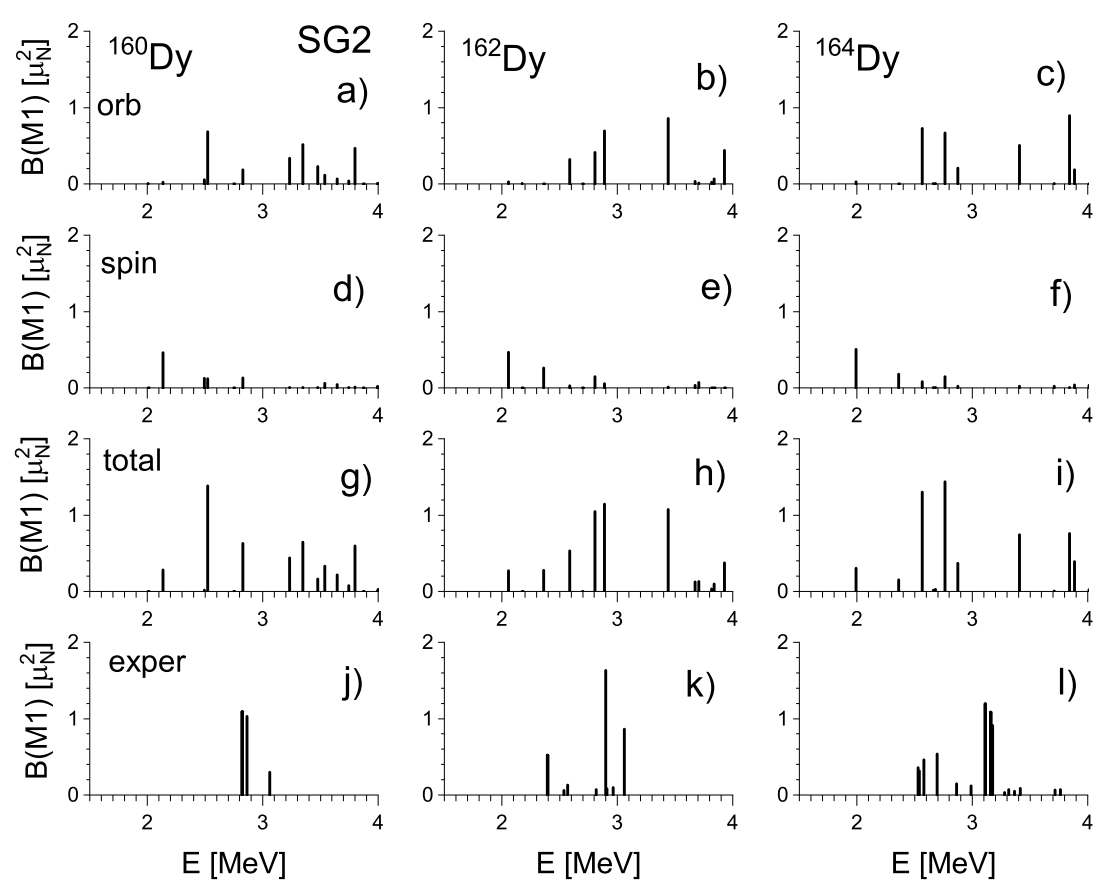

FIG. 3: Orbital (a,b,c), spin (d,e,f) and total (g,h,i) low-energy M1 strength in ${ }^{160,162,164}$ Dy, calculated in QRPA with Skyrme force SG2. In the bottom panels, the experimental M1 strength for ${ }^{160} \mathrm{Dy}$ [30] and ${ }^{162,164}$ Dy [31] is shown.

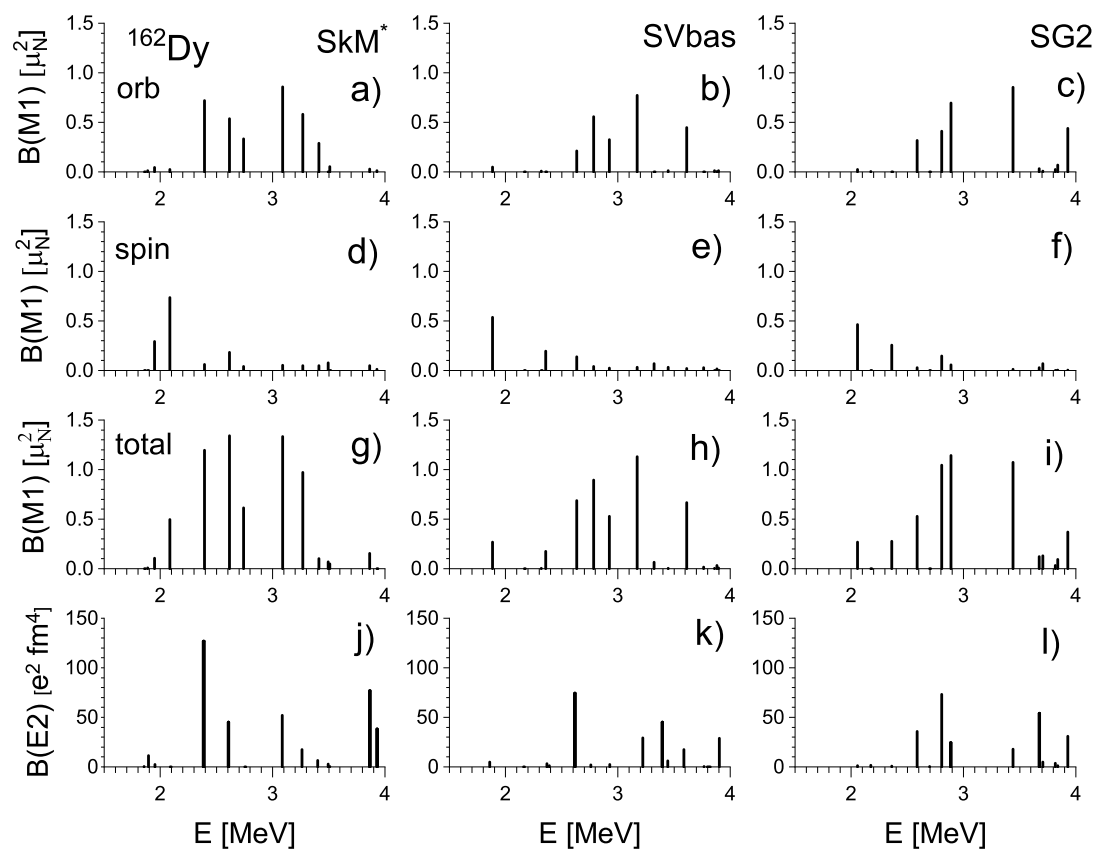

FIG. 4: Orbital (a,b,c), spin (d,e,f) and total (g,h,i) low-energy M1 strength in ${ }^{162}$ Dy, calculated in QRPA with Skyrme forces $\mathrm{SkM}^{*}$ (left), SVbas (middle) and SG2 (right). In the bottom panels, the quadrupole E2 strength is shown.

OSR states are mixtures of $M 1(K=1)$ and $E 2(K=1)$ modes, which is common in well deformed nuclei.

Note that, in WFM calculations for ${ }^{164}$ Dy 27 29], the lowest $K^{\pi}=1^{+}$state at $1.47 \mathrm{MeV}$ has a huge quadrupole strength $B(E 2)=25.44$ W.u. $\quad\left(\approx 1300 \mathrm{e}^{2}\right.$ $\mathrm{fm}^{4}$ ). The authors do not explain origin of this state.
Besides, for the next state at $2.20 \mathrm{MeV}$, the plots (ab) of fig. 9 in Ref. 28] show a spurious-like isoscalar flow. By our opinion, the $1.47-\mathrm{MeV}$ state is spurious, and higher states can also have spurious admixtures despite the statements [27 29] that spurious modes are extracted in WFM by construction. Note that similar lowest-by- 
TABLE IV: The calculated orbital, spin and total strengths $\sum B(M 1)$ (in $\left.\mu_{N}^{2}\right)$ in ${ }^{160,162,164} \mathrm{Dy}$, summed at SSR (0-2.4 MeV), OSR (2.4-4 MeV) and total (0-4 MeV) energy ranges as compared with experimental data for ${ }^{160} \mathrm{Dy}\left[30\right.$ ] and ${ }^{162,164} \mathrm{Dy}[31$ ]. For each energy range, the interference factors $R$ are shown.

\begin{tabular}{|c|c|c|c|c|c|c|c|c|c|c|c|c|c|c|}
\hline \multirow[t]{3}{*}{ Nucleus } & \multirow[t]{3}{*}{ Force } & \multicolumn{4}{|c|}{$0-2.4 \mathrm{MeV}$} & \multicolumn{4}{|c|}{ 2.4-4 MeV } & \multicolumn{5}{|c|}{$0-4 \mathrm{MeV}$} \\
\hline & & \multicolumn{3}{|c|}{$\sum B(M 1)$} & \multirow[t]{2}{*}{$\mathrm{R}$} & \multicolumn{3}{|c|}{$\sum B(M 1)$} & \multirow[t]{2}{*}{$\mathrm{R}$} & \multicolumn{4}{|c|}{$\sum B(M 1)$} & \multirow[t]{2}{*}{$\mathrm{R}$} \\
\hline & & orb & spin & total & & orb & spin & total & & orb & spin & total & $\exp$ & \\
\hline \multirow{3}{*}{${ }^{160} \mathrm{Dy}$} & $\mathrm{SkM}^{*}$ & 0.52 & 0.96 & 1.32 & 0.89 & 2.79 & 0.55 & 4.85 & 1.45 & 3.31 & 1.51 & 6.16 & & 1.28 \\
\hline & SVbas & 0.05 & 0.49 & 0.23 & 0.43 & 2.15 & 0.51 & 3.80 & 1.43 & 2.20 & 1.00 & 4.03 & 2.42 & 1.26 \\
\hline & $\mathrm{SG}_{2}$ & 0.03 & 0.46 & 0.28 & 0.57 & 2.69 & 0.54 & 4.53 & 1.40 & 2.72 & 1.00 & 4.81 & & 1.29 \\
\hline \multirow{3}{*}{${ }^{162}$ Dy } & $\mathrm{SkM}^{*}$ & 0.80 & 1.09 & 1.80 & 0.95 & 2.69 & 0.51 & 4.63 & 1.45 & 3.49 & 1.60 & 6.44 & & 1.27 \\
\hline & SVbas & 0.06 & 0.73 & 0.45 & 0.57 & 2.35 & 0.40 & 4.04 & 1.47 & 2.41 & 1.14 & 4.49 & 3.45 & 1.26 \\
\hline & $\mathrm{SG} 2$ & 0.03 & 0.72 & 0.55 & 0.73 & 2.85 & 0.35 & 4.54 & 1.42 & 2.88 & 1.07 & 5.09 & & 1.29 \\
\hline \multirow{3}{*}{${ }^{164}$ Dy } & $\mathrm{SkM}^{*}$ & 0.96 & 1.09 & 2.11 & 1.03 & 2.18 & 0.40 & 3.94 & 1.53 & 3.14 & 1.49 & 6.05 & & 1.31 \\
\hline & SVbas & 0.06 & 0.63 & 0.32 & 0.47 & 2.52 & 0.50 & 4.37 & 1.45 & 2.57 & 1.13 & 4.69 & 6.17 & 1.27 \\
\hline & SG2 & 0.03 & 0.68 & 0.45 & 0.63 & 3.20 & 0.35 & 5.05 & 1.42 & 3.23 & 1.03 & 5.50 & & 1.29 \\
\hline
\end{tabular}

energy spurious states appear in QRPA calculations if the 2qp basis is insufficient and/or the procedure for removal of spurious states is not exact. Our QRPA calculations for ${ }^{160,162,164}$ Dy (with accurate extraction of spurious admixtures by method [44]) do not give lowenergy $K^{\pi}=1^{+}$states with so high $B(E 2)$, see e.g. Fig. 4. Moreover, such states are not known experimentally and, to our knowledge, absent in other microscopic calculations, see e.g. Ref. [53] for ${ }^{164} \mathrm{Dy}$.

In Table IV, we show spin, orbital, and total QRPA strengths $\sum B(M 1)$ summed in the SSR $(0-2.4 \mathrm{MeV})$, OSR $(2.4-4 \mathrm{MeV})$ and SSR+OSR $(0-4 \mathrm{MeV})$ energy intervals. The total QRPA strengths are compared with NRF experimental data for $1^{+}$states observed at 2.8 $3.1 \mathrm{MeV}$ in $\left.{ }^{160} \mathrm{Dy}[30]\right), 2.3-3.1 \mathrm{MeV}$ in ${ }^{162} \mathrm{Dy}$ [31] and $2.5-3.8 \mathrm{MeV}$ in ${ }^{164} \mathrm{Dy}$ [31].

Table IV shows that at $0-2.4 \mathrm{MeV}$ the spin strength dominates over the orbital one. For SkM*, the orbital fraction in this interval is also essential. In OSR region 2.4 - $4 \mathrm{MeV}$, the orbital $M 1$ strength strongly dominates though the spin strength is large as well.

Following Table [V] QRPA total M1 strengths summed at $0-4 \mathrm{MeV}$ significantly overestimate the experimental values in ${ }^{160,162}$ Dy but generally correspond to the experiment in ${ }^{164} \mathrm{Dy}$ (SkM* and SG2). Perhaps, as mentioned above, the experimental data for ${ }^{160,162} \mathrm{Dy}$ [30, 31] miss a significant part of $M 1$ strength. Also, the present calculations do not take into account a coupling with complex configurations which can spread the strength and so decrease $\sum B(M 1)$-values at $0-4 \mathrm{MeV}$. Our results significantly depend on the applied Skyrme force. For example, in all considered nuclei, SVbas gives much smaller orbital and total strengths than $\mathrm{SkM}^{*}$ and SG2. This can be explained by a stronger pairing in SVbas (see discussion of Table III in Sec. II), which upshifts a part of $M 1$ strength above $4 \mathrm{MeV}$.

In both SSR and OSR regions, we see an interference between spin and orbital contributions to the total strength (i.e. the sum of spin and orbital contributions does not equal to the total strength). It is convenient to estimate this effect by an interference factor

$$
R=\frac{\sum B(M 1)_{t}}{\sum B(M 1)_{o}+\sum B(M 1)_{s}}
$$

where $\sum B(M 1)_{o}, \quad \sum B(M 1)_{s}$ and $\sum B(M 1)_{t}$ are summed orbital, spin and total strengths. The interference is destructive at $R<1$, constructive at $R>1$ and absent at $R=1$.

Table IV shows that the interference is destructive in SSR range (with exception of $\mathrm{SkM}^{*}$ case in ${ }^{164} \mathrm{Dy}$ ) and constructive in OSR range. The interference greatly increases the role of the minor spin fraction in the OSR range. For example, in ${ }^{162}$ Dy (SG2), the interference results in the total strength $4.54 \mu_{N}^{2}$ which is much larger than the orbital strength $2.85 \mu_{N}^{2}$.

Our results generally agree with the study of lowenergy $(0-4 \mathrm{MeV}) K^{\pi}=1^{+}$states in ${ }^{160,162,164}$ Dy, performed within the Quasiparticle-Phonon Nuclear Model (QPNM) [53]. This model is not self-consistent. However it has an advantage of taking into account the coupling with complex configurations. In agreement with our results, QPNM also predicts in Dy isotopes a well separated group of $1^{+}$states located at $2-2.6 \mathrm{MeV}$ and carrying a noticeable fraction of spin M1 strength. However, in QPNM the total strength of these states is mainly orbital. Only in two states at $2.0-2.1 \mathrm{MeV}$ in ${ }^{164} \mathrm{Dy}$ spin contribution to $M 1$ strength dominates over the orbital one. Coupling with complex configurations is found strong in OSR region and weaker for lower excitations. This effect can additionally downshift the orbital strength to the lower SSR region. QPNM also predicts a considerable interference between spin and orbital contributions.

For a better understanding of our results, it is worth to consider the structure and other features of the most interesting $1^{+}$states. They are shown for ${ }^{162}$ Dy in Table V] We present two states with the largest spin strength $B(M 1)_{s}$ and one state with the largest orbital strength $B(M 1)_{o}$. In the spin states, we have $B(M 1)_{s}>B(M 1)_{o}$. 
TABLE V: Characteristics of some relevant low-energy $K_{\nu}^{\pi}=1_{\nu}^{+}$states in ${ }^{162}$ Dy, calculated within QRPA with the forces $\mathrm{SkM}^{*}$, SVbas and SG2. For each state, we show the excitation energy $E$, orbital, spin and total reduced transition probabilities $B(M 1)$ and main 2qp components (contribution to the state norm in \%, structure in terms of Nilsson asymptotic quantum numbers, position of the involved single-particle states relative to the Fermi level F, and original quantum subshells in the spherical limit).

\begin{tabular}{|c|c|c|c|c|c|c|c|c|c|}
\hline \multirow[t]{2}{*}{ Force } & \multirow[t]{2}{*}{$\nu$} & \multirow{2}{*}{$\frac{\mathrm{E}}{[\mathrm{MeV}]}$} & \multicolumn{3}{|c|}{$B(M 1)\left[\mu_{N}^{2}\right]$} & \multicolumn{4}{|c|}{ main 2qp components } \\
\hline & & & orb & spin & total & $\%$ & {$\left[N, n_{z}, \Lambda\right]$} & F-position & spher. limit \\
\hline \multirow[t]{6}{*}{$\mathrm{SkM}^{*}$} & 3 & 1.95 & 0.05 & 0.29 & 0.11 & 69 & $\operatorname{pp}[411 \uparrow, 411 \downarrow]$ & $F-1, F+1$ & $2 d_{5 / 2}, 2 d_{3 / 2}$ \\
\hline & & & & & & 30 & nn $[521 \uparrow, 521 \downarrow]$ & $F-1, F+2$ & $2 f_{7 / 2}, 2 f_{5 / 2}$ \\
\hline & 4 & 2.08 & 0.02 & 0.73 & 0.50 & 69 & nn $[521 \uparrow, 521 \downarrow]$ & $F-1, F+2$ & $2 f_{7 / 2}, 2 f_{5 / 2}$ \\
\hline & & & & & & 28 & $\operatorname{pp}[411 \uparrow, 411 \downarrow]$ & $F-1, F+1$ & $2 d_{5 / 2}, 2 d_{3 / 2}$ \\
\hline & 8 & 3.09 & 0.86 & 0.05 & 1.33 & 61 & $\mathrm{nn}[521 \uparrow, 512 \uparrow]$ & $F-1, F+4$ & $2 f_{7 / 2}, 2 f_{7 / 2}$ \\
\hline & & & & & & 25 & $\operatorname{pp}[411 \uparrow, 402 \uparrow]$ & $F-1, F+4$ & $2 d_{5 / 2}, 2 d_{5 / 2}$ \\
\hline \multirow[t]{6}{*}{ SVbas } & 1 & 1.88 & 0.05 & 0.54 & 0.27 & 97 & $\operatorname{pp}[411 \uparrow, 411 \downarrow]$ & $F, F+1$ & $2 d_{5 / 2}, 2 d_{3 / 2}$ \\
\hline & & & & & & 2 & $\mathrm{nn}[521 \uparrow, 521 \downarrow]$ & $F-1, F+2$ & $2 f_{7 / 2}, 2 f_{5 / 2}$ \\
\hline & 4 & 2.36 & $\sim 0$ & 0.20 & 0.18 & 94 & $\mathrm{nn}[521 \uparrow, 521 \downarrow]$ & $F-1, F+2$ & $2 f_{7 / 2}, 2 f_{5 / 2}$ \\
\hline & & & & & & 2 & $\operatorname{pp}[411 \uparrow, 411 \downarrow]$ & $F, F+1$ & $2 d_{5 / 2}, 2 d_{3 / 2}$ \\
\hline & 8 & 3.17 & 0.77 & 0.04 & 1.13 & 65 & nn $[521 \uparrow, 512 \uparrow]$ & $F-1, F+4$ & $2 f_{7 / 2}, 1 h_{9 / 2}$ \\
\hline & & & & & & 16 & pp $[413 \downarrow, 404 \downarrow]$ & $F-2, F+4$ & $1 g_{7 / 2}, 1 g_{7 / 2}$ \\
\hline \multirow[t]{4}{*}{$\mathrm{SG} 2$} & 1 & 2.06 & 0.03 & 0.46 & 0.27 & 99 & $\operatorname{pp}[411 \uparrow, 411 \downarrow]$ & $F, F+1$ & $2 d_{5 / 2}, 2 d_{3 / 2}$ \\
\hline & 3 & 2.36 & $\sim 0$ & 0.26 & 0.28 & 99 & $\mathrm{nn}[521 \uparrow, 521 \downarrow]$ & $F-1, F+2$ & $2 f_{7 / 2}, 2 f_{5 / 2}$ \\
\hline & 8 & 3.44 & 0.86 & 0.01 & 1.07 & 57 & $\mathrm{nn}[521 \uparrow, 512 \uparrow]$ & $F-1, F+4$ & $2 f_{7 / 2}, 1 h_{9 / 2}$ \\
\hline & & & & & & 31 & pp $[413 \downarrow, 404 \downarrow]$ & $F-2, F+4$ & $1 g_{7 / 2}, 1 g_{7 / 2}$ \\
\hline
\end{tabular}

Their main 2qp components, proton [411 $\uparrow, 411 \downarrow]$ and neutron $[521 \uparrow, 521 \downarrow]$, are of the spin-flip character and correspond to particle-hole (1ph) transitions. Note that the same spin-flip 2qp configurations were found in QPNM calculations [53] for low-energy $1^{+}$states Dy isotopes. In the spherical limit, these configurations are reduced to spin-flip partners $2 d_{5 / 2}, 2 d_{3 / 2}$ and $2 f_{7 / 2}, 2 f_{5 / 2}$ with low orbital moments $l=2$ and 3 . For low $l$, the spinorbit energy splitting $\sim(\mathbf{l} \cdot \mathbf{s})$ is small and leads to lowenergy spin-flip excitations. The states with larger $l$ contribute to the spin-flip giant resonance located at a higher energy. Altogether, we see that so called SSR states are actually ordinary low-energy non-collective spin-flip excitations.

The orbital and spin-flip $M 1$ transitions in ${ }^{162}$ Dy can be illustrated using neutron and proton single-particle level schemes. In Fig. 5, we show a proton scheme for $2 d$ subshell, calculated with SG2 at the equilibrium deformation $\beta=0.346$. This scheme demonstrates the same physical mechanisms as in Fig. 2 but now for the case including the proton spin-flip transition $3 / 2^{+}[411 \uparrow$ ]$\rightarrow 1 / 2^{+}[411 \downarrow]$ which is of our interest. We see that the low-energy spin-flip transition $2 d_{5 / 2} \rightarrow 2 d_{3 / 2}$ can take place already in the spherical case. In the deformed case, two spin-flip and three orbital M1 transitions are possible. However, only two of these transitions are of $1 p h$ character and so not suppressed (other transitions can appear only due to the pairing). They are spin-flip $3 / 2^{+}\left[\begin{array}{ll}411 & \uparrow\end{array}\right] \rightarrow 1 / 2^{+}\left[\begin{array}{cl}411 & \downarrow\end{array}\right]$ and orbital $3 / 2^{+}[411 \uparrow] \rightarrow 5 / 2^{+}[402 \uparrow]$. As seen from Table $\mathbf{V}$ the

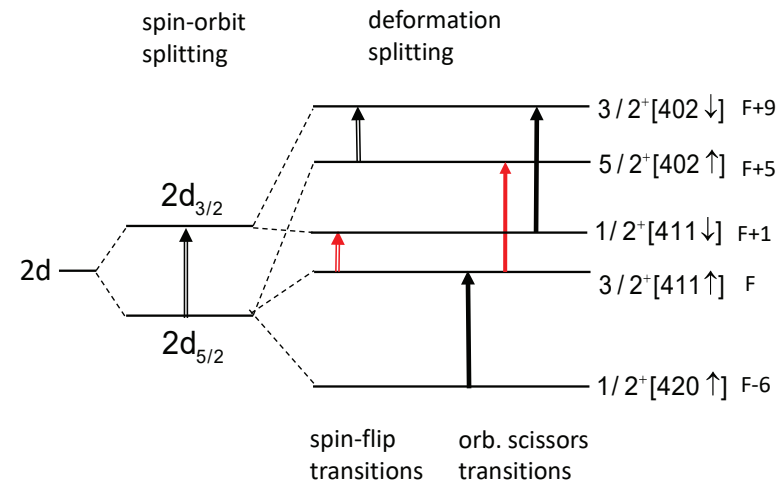

FIG. 5: A calculated (SG2) scheme of spin-flip (left empty arrows) and orbital scissors (right filled arrows) $M 1$ transitions in the proton $2 d$ subshell in ${ }^{162}$ Dy. As indicated in the top inscriptions, the left part of the figure demonstrates a spinorbit splitting into $2 d_{3 / 2}$ and $2 d_{5 / 2}$ levels in the spherical case, while the right part exhibits an additional deformation splitting. In the deformed case, $M 1(\Delta K)=1$ transitions form two groups, spin-flip and orbital scissors, as indicated in the bottom inscriptions. The Fermi level is $3 / 2^{+}[411 \uparrow]$. The $1 p h$ transitions, spin-flip $3 / 2^{+}[411 \uparrow] \rightarrow 1 / 2^{+}[411 \downarrow]$ and orbital $3 / 2^{+}[411 \uparrow] \rightarrow 5 / 2^{+}[402 \uparrow]$, are marked by red color.

proton spin-flip 2qp configuration [411 个, $411 \downarrow$ ] indeed dominates in the states at $1.95 \mathrm{MeV}\left(\mathrm{SkM}^{*}\right), 1.88 \mathrm{MeV}$ (SVbas), and $2.06 \mathrm{MeV}$ (SG2). The orbital configuration $[411 \uparrow, 402 \uparrow]$ is fragmented between many states, it is seen e.g. in $3.09-\mathrm{MeV}$ state $\left(\mathrm{SkM}^{*}\right)$. Since deforma- 


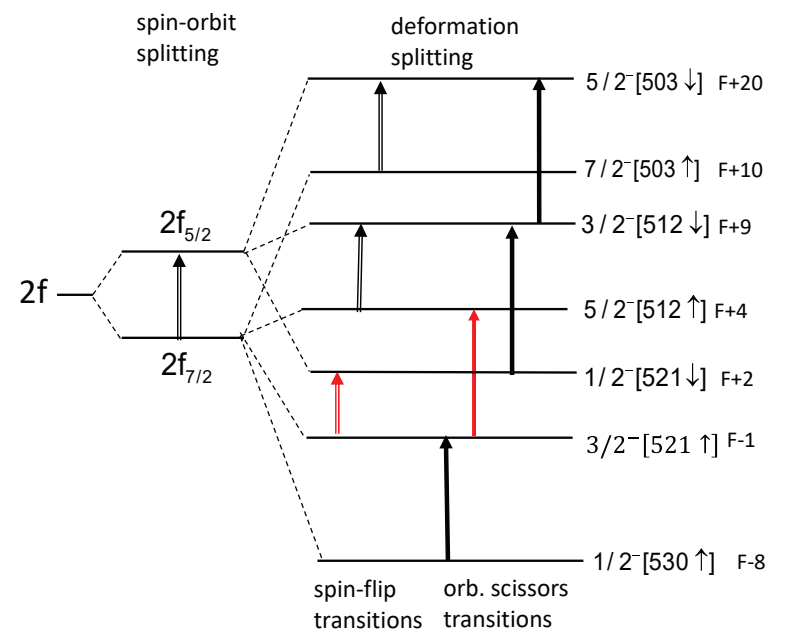

FIG. 6: The same as in Fig. 5 but for the neutron $2 f$ subshell in ${ }^{162}$ Dy. The $1 p h$ spin-flip $3 / 2^{-}[521 \uparrow] \rightarrow 1 / 2^{-}[521 \downarrow]$ and orbital $3 / 2^{-}[521 \uparrow] \rightarrow 5 / 2^{-}[512 \uparrow]$ transitions are marked by red color.

tions in ${ }^{160,162,164}$ Dy are similar (see Table II), the same results should take place for ${ }^{160}$ Dy and ${ }^{164}$ Dy as well.

A similar analysis can be done for a neutron singleparticle scheme in ${ }^{162} \mathrm{Dy}$. A relevant part of this scheme for $2 f$ subshell is shown in Fig. 6. We see that again, among many possible spin-flip and orbital $M 1$ transitions, there are only two $1 p h$ transitions: spin-flip $3 / 2^{-}[521 \uparrow] \rightarrow 1 / 2^{-}[521 \downarrow]$ and orbital $3 / 2^{-}[521 \uparrow] \rightarrow$ $5 / 2^{-}[512 \uparrow]$. The corresponding 2 qp configurations indeed take place in Table $\mathrm{V}$.

It is easy to recognize from Fig. 6 that ${ }^{160} \mathrm{Dy}$ and ${ }^{164}$ Dy, whose Fermi levels correspond to $F-1$ and $F+1$ states of the given neutron scheme, also allow $1 \mathrm{ph}$ spinflip transitions $3 / 2^{-}[521 \uparrow] \rightarrow 1 / 2^{-}[521 \downarrow]$. This explains why in our calculations all three isotopes ${ }^{160,162,164} \mathrm{Dy}$ demonstrate similar distributions of low-lying spin-flip excitations.

\section{B. Nuclear currents in ${ }^{162}$ Dy}

In this section, we show various $\mathrm{CTD} \delta \mathbf{j}_{\nu}(\mathbf{r})$ defined in Sec. II. CTD are calculated with the force SG2 for a few relevant states in ${ }^{162}$ Dy, shown in Table V First, we consider 3.44-MeV state which, following Fig. 4, demonstrates the largest orbital M1 strength. Figure 7 shows for this state the proton, neutron, isoscalar $(\Delta \mathrm{T}=0)$, and isovector $(\Delta \mathrm{T}=1) \mathrm{CTD}$ plotted on $(x, z)$-plane, where $z$ is the nuclear symmetry axis. Magnitudes of the currents are equally scaled to provide distinctive pictures. So, only relative lengths of the current arrows and their directions (but not absolute lengths of arrows) are matter. The nuclear boundary estimated for the sharp nucleus edge is depicted by a solid ellipse.

Figure 7 shows that protons and neutrons in $3.44-\mathrm{MeV}$ state move in opposite directions at the left and right

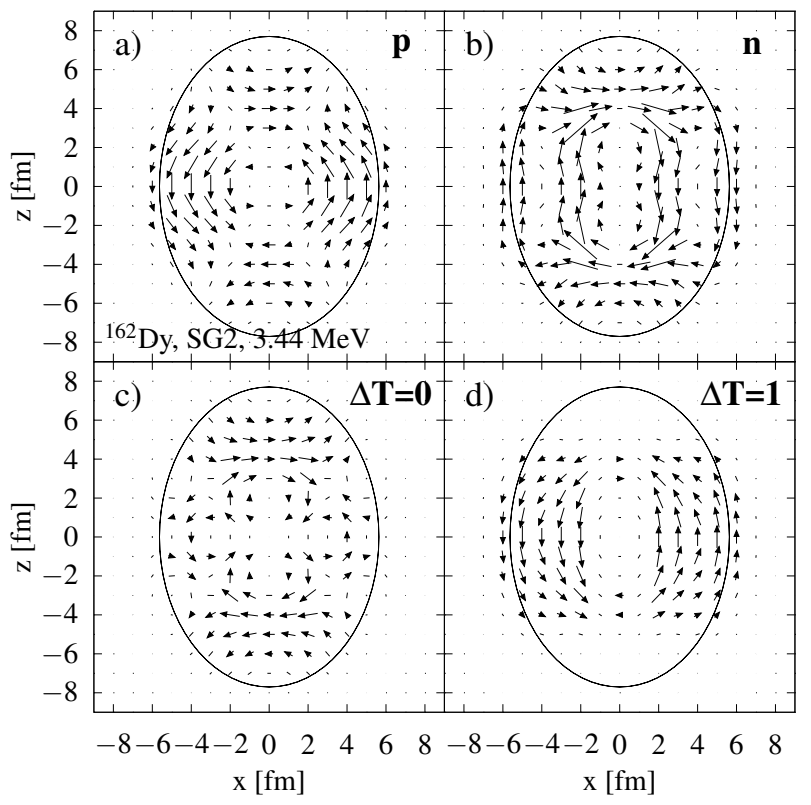

FIG. 7: Proton (a), neutron (b), isoscalar (c), and isovector $(\mathrm{d})$ convection CTD in $(x, z)$ plane for $3.44-\mathrm{MeV}$ state in ${ }^{162}$ Dy, calculated within QRPA with the force SG2. A solid ellipse shows the nuclear boundary.

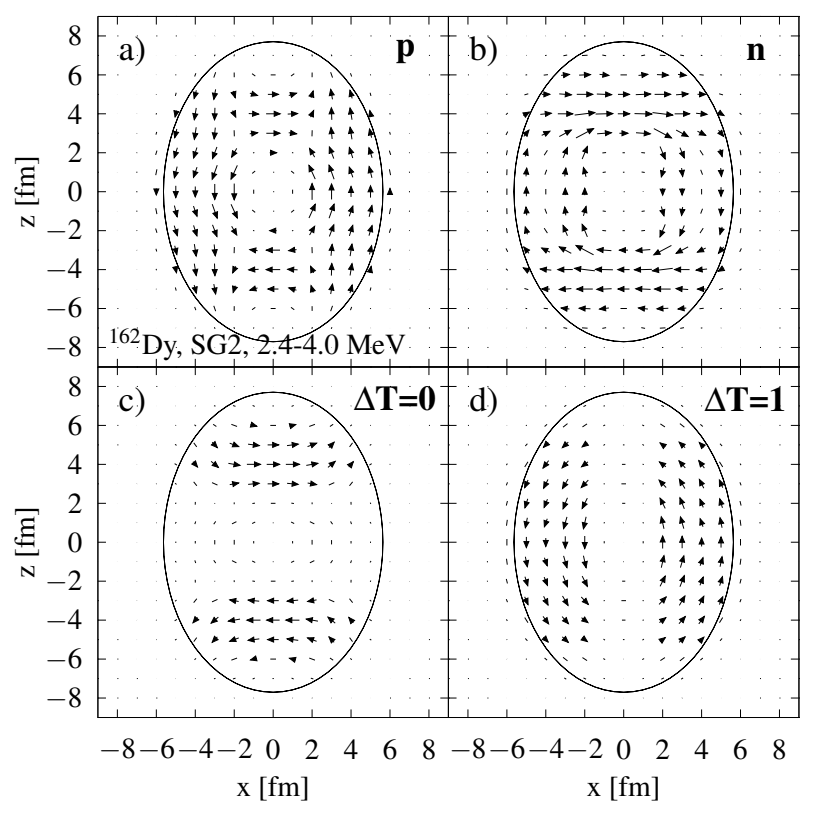

FIG. 8: The same as in Fig. 7 but for the energy interval 2.4-4 MeV.

surface regions (cf. plots a), b) and d)) and this motion resembles an isovector OSR (a similar orbital current was earlier obtained in deformed ${ }^{50} \mathrm{Cr}$ [21]). Following Table V] 3.44-MeV state has large proton (57\%) and neutron (31\%) 2qp components. This complicates a general flow and makes it different (in the pole regions) from the simple collective OSR picture. We also see that $3.44-\mathrm{MeV}$ state exhibits both isoscalar and isovector currents. 


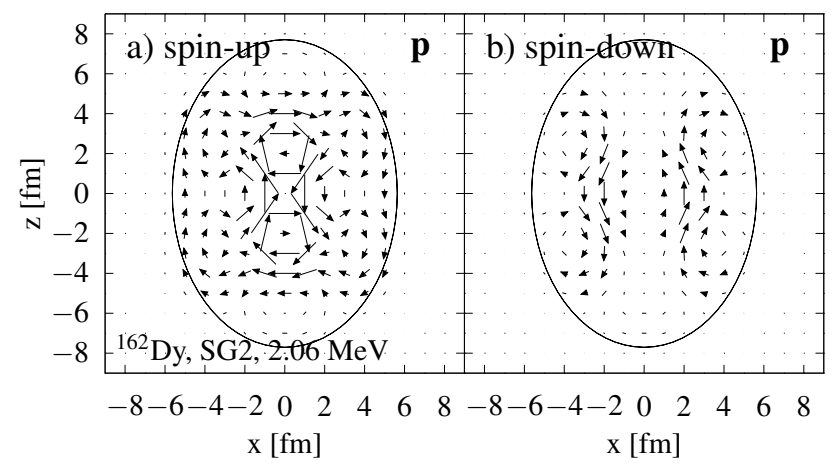

FIG. 9: Proton spin-up (a) and spin-down (b) CTD in basically proton $2.06-\mathrm{MeV}$ state in ${ }^{162} \mathrm{Dy}$.

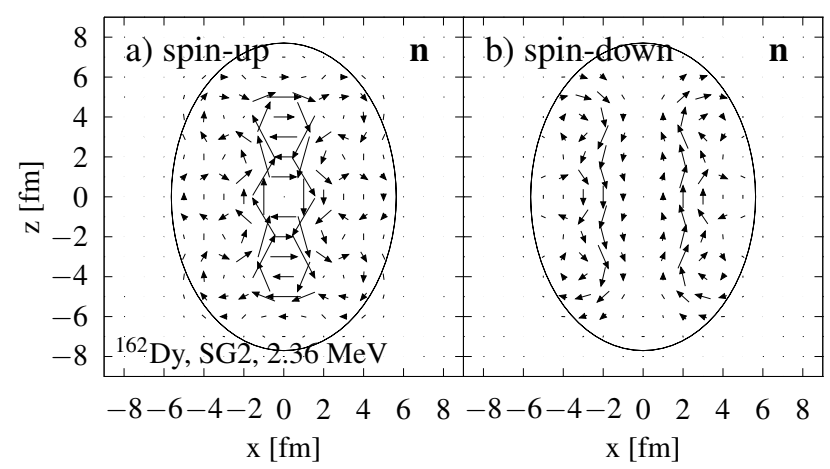

FIG. 10: The same as in Fig. 9 but for CTD in basically neutron $2.36-\mathrm{MeV}$ state in ${ }^{162} \mathrm{Dy}$.

For a reasonable comparison with collective WFM currents, it is worth to consider the summed CTD involving contributions of all QRPA states from the OSR energy range 2.4-4 MeV. The summed CTD will smooth individual peculiarities of the currents of particular QRPA states and thus highlight the main (e.g. collective) features of the nuclear flow in the given energy range. The procedure to get summed CTD is described in Ref. [57]. The summed CTD are shown in Fig. 8. The flow in left/right surface regions now more resembles the OSR picture. However, the flow is again mixed by isospin. It is isovector in the left/right sides and isoscalar in the pole regions.

Following WFM 28, 29], the low-energy spin states should demonstrate out-of-phase rotation-like oscillations of spin-up and spin-down nuclear fractions, see Fig. $1 \mathrm{~b})$. To check this prediction, we show in Figs. 9 and 10 spin-up and spin-down CTD for spin-flip states at 2.06 and $2.36 \mathrm{MeV}$. As seen from Table $\mathrm{V}$, these states are almost fully exhausted by one proton and one neutron 2qp component, respectively. So, to characterize the nuclear flow in these states, the corresponding proton and neutron spin-up and spin-down currents are enough. Figures 9 and 10 show that the currents are not regular but rather demonstrate a complex cellular-like structure formed by the dominant 2qp configurations. They are proton $[411 \uparrow, 411 \downarrow]$ and neutron $[521 \uparrow, 521 \downarrow]$ config-

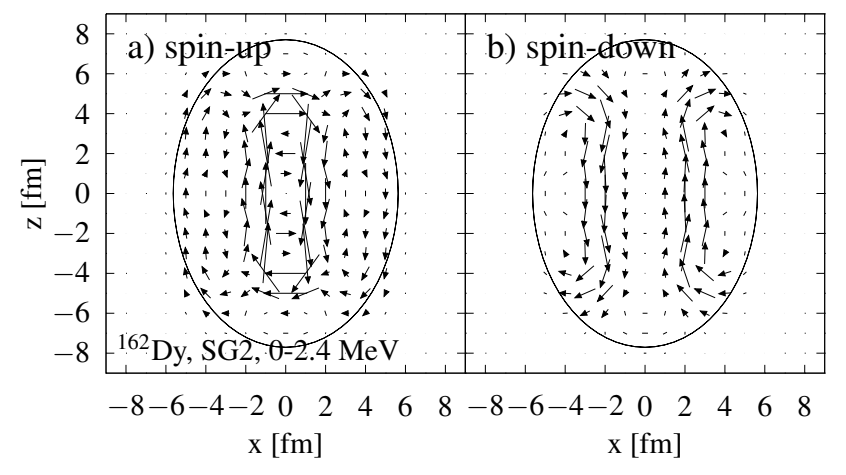

FIG. 11: Spin-up (a) and spin-down (b) CTD for the energy interval 0-2.4 MeV
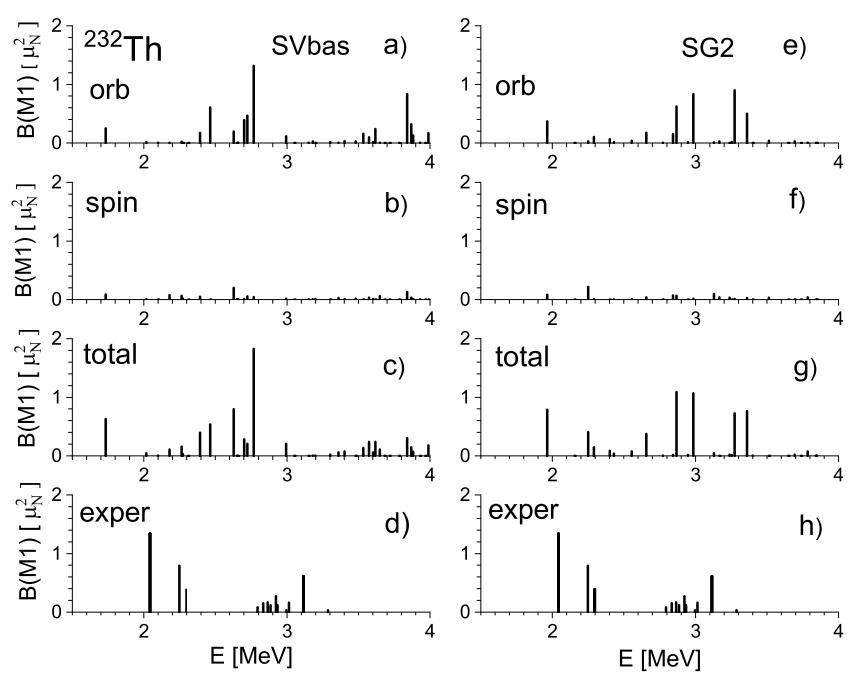

FIG. 12: The computed (SVbas, SG2) orbital, spin and total low-energy M1 strengths in ${ }^{232} \mathrm{Th}$ as compared with the experimental data 34 .

urations arising from $2 d(l=2)$ and $2 f(l=3)$ spherical subshells. Accordingly, the proton flow in Fig. 9 has a fewer number of cells than the neutron one in Fig. 10.

In Fig. 11, the summed CTD are depicted. They do not match regular collective WFM spin-scissors currents shown in Ref. [28].

\section{C. $\quad M 1$ strength in ${ }^{232} \mathrm{Th}$}

In addition to strongly deformed Dy isotopes, SSR was also predicted by WFM in a less deformed nucleus ${ }^{232} \mathrm{Th}$ [25, 26, 28]. In this nucleus, the experiment [34] also gives two separate groups of low-energy $1^{+}$states (see plot (d) in Fig. 12). The lower group at $E<2.5 \mathrm{MeV}$ is considered by WFM as a candidate for SSR. In this connection, we present QRPA results for ${ }^{232} \mathrm{Th}$, obtained with the forces SVbas and SG2. Note that these forces, especially SG2, provide a good description of the spin-flip M1 giant resonance in ${ }^{232} \mathrm{Th}$, see Appendix A.

In Fig. 12, the computed orbital, spin, and total 
TABLE VI: The same as in Table V but for states in ${ }^{232} \mathrm{Th}$.

\begin{tabular}{|c|c|c|c|c|c|c|c|c|c|}
\hline Force & $\nu$ & $\mathrm{E}$ & \multicolumn{3}{|c|}{$B(M 1)\left[\mu_{N}^{2}\right]$} & \multicolumn{4}{|c|}{ main 2qp components } \\
\hline & & {$[\mathrm{MeV}]$} & orb & spin & total & $\%$ & {$\left[N, n_{z}, \Lambda\right]$} & F-position & spher. limit \\
\hline \multirow{3}{*}{ SVbas } & 1 & 1.73 & 0.25 & 0.09 & 0.63 & 91 & $\mathrm{pp}[660 \uparrow, 651 \uparrow]$ & $F, F+1$ & $1 i_{13 / 2}, 1 i_{13 / 2}$ \\
& 15 & 2.77 & 1.32 & 0.04 & 1.82 & 51 & $\mathrm{nn}[761 \uparrow, 752 \uparrow]$ & $F-1, F-3$ & $1 j_{15 / 2}, 1 j_{15 / 2}$ \\
\hline \multirow{3}{*}{ SG2 } & 1 & 1.96 & 0.18 & 0.04 & 0.39 & 89 & $\mathrm{pp}[660 \uparrow, 651 \uparrow]$ & $F, F+1$ & $1 i_{13 / 2}, 1 i_{13 / 2}$ \\
& 3 & 2.25 & 0.01 & 0.11 & 0.20 & 96 & $\mathrm{nn}[631 \uparrow, 631 \downarrow]$ & $F, F+3$ & $1 i_{13 / 2}, 3 d_{5 / 2}$ \\
& 13 & 2.98 & 0.42 & 0.01 & 0.53 & 32 & $\mathrm{pp}[530 \uparrow, 521 \uparrow]$ & $F-1, F+4$ & $2 f_{7 / 2}, 2 f_{7 / 2}$ \\
\hline
\end{tabular}

TABLE VII: The computed orbital, spin and total $B(M 1)$ strengths summed at $\mathrm{E}=0-3.3 \mathrm{MeV}$ as compared with the experimental data [34]. $R$ are the interference factors.

\begin{tabular}{|c|c|c|c|c|c|}
\hline Force & \multicolumn{3}{|c|}{$\sum B(M 1)\left[\mu_{N}^{2}\right]$} & $\mathrm{R}$ \\
\hline & orb & spin & total & exper & \\
\hline SVbas & 3.60 & 0.66 & 5.23 & & 1.23 \\
SG2 & 3.37 & 0.68 & 4.92 & 4.26 & 1.21 \\
\hline
\end{tabular}

$B(M 1)$ strengths in ${ }^{232} \mathrm{Th}$ are compared with NRF experimental data [34]. We see that the spin strength is much smaller that the orbital one even at $E<2.5 \mathrm{MeV}$. For SG2, there is a remarkable agreement between the distribution of the total strength and the experimental data. Namely, both experiment and theory give at $E<$ $2.5 \mathrm{MeV}$ the distinctive group of the states. Fig. 12 obviously does not demonstrate any distinctive SSR. Indeed, both level groups, below and above $2.5 \mathrm{MeV}$, are strongly dominated by the orbital strength. So, these two groups are explained not by separation of SSR and OSR modes (as was suggested by WFM) but rather by a fine structure of the OSR alone.

In TableVI, we show the features of some representative states with the large spin and orbital strength. In SVbas case, the first state is not spin-flip despite it has the largest spin strength at the range $E<2.5 \mathrm{MeV}$. Moreover, it is dominated by the orbital strength. This state is not collective and demonstrates a constructive interference of the spin and orbital contributions, in contrast to the lowest states in Dy isotopes. The $2.77-\mathrm{MeV}$ state is collective and exhibits a constructive interference like the orbital states in Dy case. In SG2 case, the first $1.96-\mathrm{MeV}$ state is non-collective and mainly orbital (like for SVbas). The third 2.98-MeV state is spin-flip one with the dominant neutron configuration [631 $\uparrow, 631 \downarrow]$. Both states demonstrate a strong constructive interference of orbital and spin contributions. The $2.98-\mathrm{MeV}$ state is a collective orbital state.

The calculated and experimental summed $M 1$ strengths are compared in TableVII Like in Dy isotopes, the theoretical values of the total $\sum B(M 1)$ somewhat overestimate the experimental data. As mentioned in Sec. III-A, the overestimation can be caused by i) missing of a significant part of $M 1$ strength in the experiment and ii) neglect of the coupling with complex configura- tions. Like in Dy isotopes, we see in ${ }^{232} \mathrm{Th}$ the constructive interference of the spin and orbital contributions to the total strength.

\section{CONCLUSIONS}

The WFM prediction of a low-energy spin-scissors resonance (SSR) in deformed nuclei [23, 25 29] was analyzed in the framework of the self-consistent QRPA approach using Skyrme forces SkM*, SVbas, and SG2. The calculations were performed for deformed nuclei ${ }^{160,162,164} \mathrm{Dy}$ and ${ }^{232} \mathrm{Th}$. Two of these nuclei, ${ }^{164} \mathrm{Dy}$ and ${ }^{232} \mathrm{Th}$, were proposed by WFM as promising candidates for SSR.

The calculations have shown that, in strongly deformed nuclei like ${ }^{160,162,164}$ Dy, indeed there can exist a group of $K^{\pi}=1^{+}$spin states located at 1.5-2.4 MeV, i.e. below the conventional orbital scissor resonance (OSR). Following our analysis, these states are ordinary spin-flip excitations characterized by $M 1(\Delta K=1)$ transitions between spin-orbit partners in subshells with a low orbital momentum $l$, e.g. $2 d$ and $1 f$. Such low- $l$ spin-flip states can form a separate low-energy group if a large deformation shifts OSR to a higher energy. In our calculations, this is the case for well deformed ${ }^{160,162,164}$ Dy but not for less deformed ${ }^{232} \mathrm{Th}$.

The obtained low-energy spin states are non-collective and mainly exhausted by one 2qp spin-flip configuration. This can be explained by basically isovector character of the spin-spin residual interaction which upshifts the collectivity to higher energies. The non-collective character of low-energy spin states contradicts with the collective scissors nature of the predicted SSR. Further, the calculated distributions of nuclear currents locally resemble the OSR collective flow but not the SSR one.

Since OSR energy $E \approx 66 \delta A^{-1 / 3} \mathrm{MeV}$ falls with the mass number $\mathrm{A}$, this resonance in heavy (actinide) nuclei goes down by energy and mixes with nearby spin states. Being stronger, OSR conceals these states. So heavy deformed nuclei are not suitable to exhibit distinctive lowenergy spin states.

At the excitation energy $E<4 \mathrm{MeV}$, most of $1^{+}$states demonstrate a significant interference of spin-flip and orbital contributions to $M 1$ strength. The interference considerably increases the total $M 1$ strength in the OSR energy range. This should be taken into account while 
comparing the computed strengths with estimations derived merely for the orbital mode. A part of the orbital strength is downshifted to the region of spin states $(E \leq$ $2.4 \mathrm{MeV}$ ) and, vice versa, the OSR region hosts some spin-flip strength.

The experimental data $31-34]$ show two distinctive low-energy groups of $1^{+}$states in ${ }^{162,164} \mathrm{Dy}$ and ${ }^{232} \mathrm{Th}$. These two groups are treated by WFM as SSR and OSR. Our calculations show that lowest $1^{+}$states in $160,162,164$ Dy are indeed of spin-flip character. However they are located at $E \leq 2.4 \mathrm{MeV}$, i.e. below the observed states. So perhaps both two observed groups are produced by fragmentation of the orbital strength. This is even more the case in ${ }^{232} \mathrm{Th}$ where the low-energy spin strength is almost negligible. So, by our opinion, the available experimental data still do not confirm the existence of SSR. More definite conclusions can be drawn after further experimental and theoretical effort. Indeed, following discussion [33], a significant number of $1^{+}$states can be found below $2.7 \mathrm{MeV}$, see database [49] for candidates. As for the theory, it should take into account the coupling with complex configurations, which, in principle, can redistribute the $M 1$ strength.

The WFM scissor-like treatment of low-energy spin M1 excitations requires the nuclear deformation. In other words, spin-scissors excitations can exist only in deformed nuclei. Instead, our calculations show that lowenergy spin states arise from the spin-orbit splitting and so can exist even in spherical nuclei. So the deformation is not the origin of the low-energy spin strength but only an essential factor affecting its properties. In principle, WFM does not use any two-rotor assumption. Then, perhaps, the deformation-induced scissors-like scheme is just a poorly chosen illustration.

The spin-orbit splitting and spin-spin residual interaction are of a primary importance in the exploration of spin excitations [4, 5]. To check the accuracy of our QRPA method in description of these factors, we performed calculations for the spin-flip M1 $(K=1)$ giant resonance in ${ }^{162} \mathrm{Dy}$ and ${ }^{232} \mathrm{Th}$ and obtained for the forces SVbas and SG2 a good agreement with the experiment. The same test should be done by WFM as well.

In WFM calculations [27 29], the lowest $K^{\pi}=1^{+}$ state with the energy $\mathrm{E}=1.47 \mathrm{MeV}$ has a huge quadrupole strength $\mathrm{B}(\mathrm{E} 2)=25.4 \mathrm{~W} . \mathrm{u}$.. The authors do not explain the origin of such state. By our opinion, this state is spurious. Neither experimental data, nor our QRPA calculations for ${ }^{160,162,164} \mathrm{Dy}$ and ${ }^{232} \mathrm{Th}$ give at $\mathrm{E}<4 \mathrm{MeV}$ $1^{+}$states with so large B(E2) value.

The discrepancy between WFM and QRPA predictions for spin states in ${ }^{232} \mathrm{Th}$ could be clarified by $\left(p, p^{\prime}\right)$ measurements which are sensitive to spin-flip excitations and not so much to orbital ones. If low-energy spin states indeed exist in ${ }^{232} \mathrm{Th}$, they should be observed in $\left(p, p^{\prime}\right)$ reaction.

Since low-energy spin states are reduced to almost pure 2qp excitations, these states can be useful for investigation of low- $l$ spin-orbit splitting and its interplay with nuclear deformation. Besides, such states can be also useful for testing tensor forces.

\section{Acknowledgement}

We thank Profs. P.-G. Reinhard, P. von NeumannCosel and A.V. Sushkov for useful discussions. The work was partly supported by Votruba - Blokhintsev (Czech Republic - BLTP JINR) grant (VON and JK) and a grant of the Czech Science Agency, Project No. 19-14048S (JK). VON and WK appreciate the Heisenberg-Landau grant (Germany - BLTP JINR). A.R. acknowlegdes the support by the Slovak Research and Development Agency under contract No. APVV-15-0225, Slovak grant agency VEGA (contract No. 2/0067/21), and the Research and Development Operational Programme funded by the European Regional Development Fund, project No. ITMS code 26210120023.

\section{Appendix A: $M 1$ spin-flip giant resonance}

Energy and structure of $M 1$ spin-flip giant resonance in open-shell nuclei are basically determined by the interplay between spin-orbital splitting in proton and neutron schemes from one side and spin-spin residual interaction from another side [2, 4, 5]. To check the accuracy of our approach, we present here QRPA results for spinflip giant resonance in ${ }^{162} \mathrm{Dy}$ and ${ }^{232} \mathrm{Th}$, obtained with the Skyrme parameterizations SkM*, SVbas, and SG2. We were not able to find experimental data for this resonance in ${ }^{162} \mathrm{Dy}$. So, for this nucleus, we compare QRPA results with the $\left(p, p^{\prime}\right)$ data for the neighbouring nucleus ${ }^{158} \mathrm{Gd}[54]$ which has a similar quadrupole deformations $\left(\beta_{2}=0.348\right)$ [49]. For ${ }^{232} \mathrm{Th}$, we use $\left(p, p^{\prime}\right)$ data [47, 55].

In Fig. 13, the results of our calculations are compared with the experimental data. QRPA strength functions are obtained by averaging transition rates $B_{\nu}(M 1)$ for separate QRPA states by Lorentz weight with an averaging parameter $\Delta=1 \mathrm{MeV}$, see Refs. [4, 5] for more detail. Only spin part of M1 transition operator (3) is used. The experimental data (in arbitrary units) are properly scaled for a convenient comparison with QRPA strength functions. Fig. 13 shows that SVbas, and especially SG2, well describe localization and fine structure of the resonance in both nuclei. In $\mathrm{SkM}^{*}$, distribution of the strength is too wide and upshifted to higher energies. This difference can be explained by smaller values of spin-flip parameters $b_{4}$ and $b_{4}^{\prime}$ in SVbas and SG2 sets (see Table I in Sec. II).

In Table VIII, the spin $B(M 1)$-values summed at the energy interval $\mathrm{E}=0-12 \mathrm{MeV}$ are compared with early QRPA results of P. Sarriguren et al [47], obtained with the force SG2. It is seen that the agreement is fine for SG2, acceptable for SVbas and worse for SkM*.

Altogether, Fig. 13 and Table VIII show that forces 

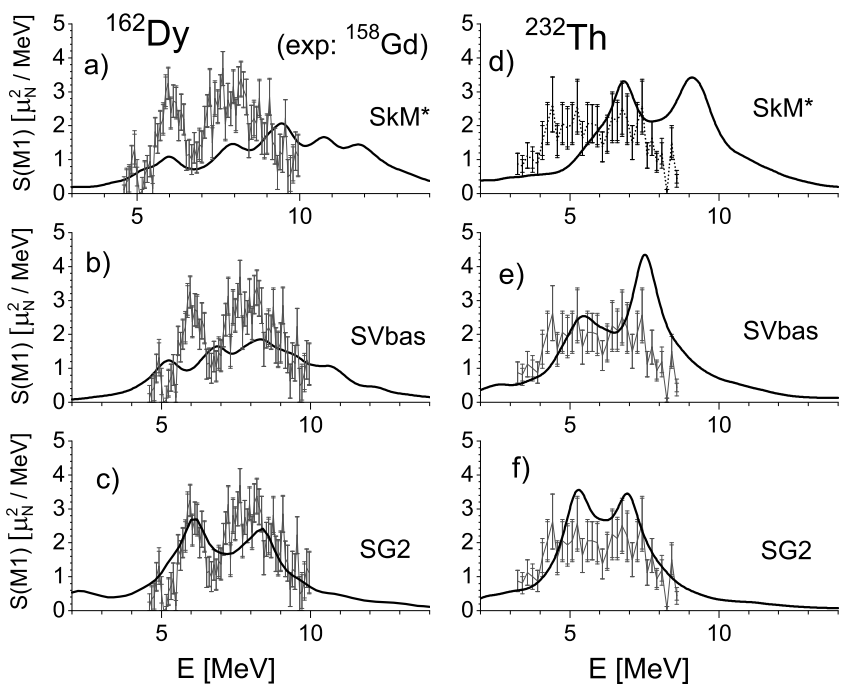

FIG. 13: $M 1$ spin-flip giant resonance in ${ }^{162} \mathrm{Dy}$ and ${ }^{232} \mathrm{Th}$, calculated with Skyrme forces SkM*, SVbas and SG2. The results are compared with scaled experimental data (in arbitrary units) for ${ }^{232} \mathrm{Th}$ [47, 55] (right plots) and neighbouring nucleus ${ }^{158} \mathrm{Gd}$ [54] (left plots). See details in the text.

TABLE VIII: The strength $B(M 1)_{s}$ summed at $\mathrm{E}=0-12 \mathrm{MeV}$ in our SkM*, SVbas and SG2 calculations as compared with QRPA (SG2) results of Sarriguren et al [47].

\begin{tabular}{|c|c|c|c|c|}
\hline Nucleus & \multicolumn{4}{|c|}{$\sum B(M 1)_{s}\left[\mu_{N}^{2}\right]$} \\
\hline & SkM$^{*}$ & SVbas & SG2 & Sarriguren [47] \\
\hline${ }^{160} \mathrm{Dy}$ & 14.5 & 13.2 & 12.4 & 11.4 \\
\hline${ }^{162} \mathrm{Dy}$ & 14.7 & 13.4 & 12.7 & 12.2 \\
\hline${ }^{164} \mathrm{Dy}$ & 14.7 & 13.6 & 12.9 & 12.2 \\
\hline${ }^{232} \mathrm{Th}$ & 17.2 & 15.9 & 14.3 & 14.9 \\
\hline
\end{tabular}

SVbas and SG2 are most relevant for exploration of spinflip excitations.

\section{Appendix B: WFM vs QRPA results}

In this Appendix, we briefly discuss some important points concerning the comparison and treatment of WFM and QRPA results.

It is known that macroscopic and microscopic models often successfully supplement each other in description of nuclear modes [1, 35]. For example, our QRPA results for isovector E1 giant resonance [56], E1 toroidal mode [57, 58] and M1 orbital scissors (present calculations) well agree with predictions of macroscopic models [59, 60], [61], and [10, 15, 16], respectively. However, we were not able to get a similar correspondence between our QRPA results and WFM predictions for SSR. In this connection, it is worth to discuss some important issues.

1) Accuracy of WFM numerical results for $M 1$ lowenergy spin states.

Both Skyrme QRPA and WFM have spin-orbit mean- field terms and so include spin-orbit splitting and corresponding spin-flip excitations. In QRPA, spin-flip states are identified by strong domination of spin-flip 2qp components, large values of spin $B(M 1)_{s}$, and hindered $B(E 2)$. WFM deals with collective variables and identifies spin states mainly by enhanced $B(M 1)$ and hindered $B(E 2)$, where $B(M 1)$ is calculated only for the total (spin+orbital) M1 operator. This seems not enough to identify reliably spin-flip states. Besides, following Table I for ${ }^{164}$ Dy in 28], WFM does not produce at all the M1 spin-flip giant resonance, which makes questionable the accuracy of WFM in description of spin-flip states. Further, the parameters of the WFM Hamiltonian (includes a spherical harmonic oscillator, spin-orbit terms, pairing, quadrupole-quadrupole and spin-spin separable residual interaction) are taken from different sources and, by our opinion, not properly justified. In this connection, the claimed good agreement of WFM results with the experimental data looks doubtful.

\section{2) Is the spin-scissors scheme generally relevant?}

The SSR macroscopic picture was suggested in analogy with OSR scheme developed within the two-rotor model [10, 15, 16]. However, the OSR scheme was confirmed by experimentally observed [13, 14] specific dependencies of OSR energy and strength on the nuclear deformation [2, 15, 16]. Instead, the WFM calculations have not still suggested any specific measurable features justifying the relevance of the spin-scissors picture.

The spin-scissors picture assumes a non-zero nuclear deformation. Without deformation this picture cannot be realized in principle. However, following our calculations, the deformation is not the primary origin of $M 1$ low-energy spin states.

In the spin-scissors picture (Fig. 1(b,c)), SSR looks as a two-step process including spin-flip excitation + orbital oscillation. It is not clear how to match such two-step process with the linear regime used in WFM. We have not found in Refs. [25-29] any relevant linear probe external field to generate such SSR.

Following Eq. (29) in Ref. [28], the WFM nuclear current is formed solely by components of an orbital collective variables for different combinations of spin directions. Maybe, for this reason, the currents for OSR, SSRI and SSR-II in Figs. 9-11 of Ref. [28] look identical (up to direction of the motion). Our QRPA distributions of the nuclear current partly support the isovector OSR scheme but not the SSR one.

Altogether, we have a feeling that the deformationinduced scissors-like picture used for illustration and interpretation of the WFM results is a poor and even misleading choice. 


\section{Appendix C: Matrix elements of magnetic transitions in axially deformed nuclei}

In cylindrical coordinates, the single-particle wave function with quantum numbers $K^{\pi}$ has the spinor form

$$
\Psi_{i}(\mathbf{r})=\left(\begin{array}{c}
R_{i}^{(+)}(\rho, z) e^{i m_{i}^{(+)} \phi} \\
R_{i}^{(-)}(\rho, z) e^{i m_{i}^{(-)} \phi}
\end{array}\right),
$$

for the normal state and

$$
\Psi_{\bar{i}}(\mathbf{r})=\hat{T} \Psi_{i}(\mathbf{r})=\left(\begin{array}{c}
-R_{i}^{(-)}(\rho, z) e^{-i m_{i}^{(-)} \phi} \\
R_{i}^{(+)}(\rho, z) e^{-i m_{i}^{(+)} \phi}
\end{array}\right)
$$

for the time-reversal state. Here the momentum projection is decomposed as $K_{i}=m_{i}^{(\sigma)}+\frac{1}{2} \sigma$ with $\sigma= \pm 1$.

The spin and orbital $M \lambda \mu$ transition operators are 62]

$$
\begin{aligned}
& \hat{S}_{l \lambda \mu}=\mu_{N} \sqrt{\lambda(2 \lambda+1)} r^{l} g_{s}^{q}\left\{\hat{\mathbf{s}} Y_{l}\right\}_{\lambda \mu}, \\
& \hat{L}_{l \lambda \mu}=\mu_{N} \sqrt{\lambda(2 \lambda+1)} r^{l} g_{l}^{q} \frac{2}{\lambda+1}\left\{\hat{\mathbf{l}} Y_{l}\right\}_{\lambda \mu}
\end{aligned}
$$

where $l=\lambda-1, \mu_{N}$ is the nuclear magneton, $\hat{\mathbf{s}}$ and $\hat{\mathbf{l}}$ are standard spin and orbital operators, $g_{s}^{q}$ and $g_{l}^{q}$ are spin and orbital gyromagnetic factors. Further

$$
\begin{aligned}
\left\{\hat{\mathbf{s}} Y_{l}\right\}_{\lambda \mu} & =\sum_{m} \sum_{\alpha=-1,0,1} C_{l m, 1 \alpha}^{\lambda \mu} Y_{l m} \hat{s}_{\alpha}, \\
\left\{\hat{\mathbf{l}} Y_{l}\right\}_{\lambda \mu} & =\sum_{m} \sum_{\alpha=-1,0,1} C_{l m, 1 \alpha}^{\lambda \mu} Y_{l m} \hat{l}_{\alpha}
\end{aligned}
$$

where $Y_{l m}$ are the spherical harmonics and $C_{l m, 1 \alpha}^{\lambda \mu}$ are Clebsch-Gordan coefficients.

The matrix elements for the orbital and spin $M \lambda \mu$ transitions from the BCS vacuum $|\mathrm{BCS}\rangle$ to the twoquasiparticle (2qp) state $\alpha_{i}^{+} \alpha_{j}^{+}|\mathrm{BCS}\rangle$ with the selection rule $\left|K_{i}-K_{j}\right|=\mu\left(K_{i}, K_{j}>0, \mu \geq 0\right)$ have the form

$$
\begin{gathered}
\left\langle i \bar{j}\left|\hat{L}_{l \lambda \mu}\right| \mathrm{BCS}\right\rangle=2 \pi \mu_{N} \sqrt{\lambda(2 \lambda+1)} \frac{2 g_{l}}{\lambda+1} u_{i j}^{(-)} \quad(\mathrm{C} 7) \\
\cdot \int d z d \rho \rho\left\{g_{l \mu} C_{l \mu, 10}^{\lambda \mu}\left[R_{i}^{(+)} m_{j}^{(+)} R_{j}^{(+)}+R_{i}^{(-)} m_{j}^{(-)} R_{j}^{(-)}\right]\right. \\
+\frac{1}{\sqrt{2}} g_{l \mu+1} C_{l \mu+1,1-1}^{\lambda \mu}\left[R_{i}^{(+)}\left(\rho \frac{d}{d z}-z \frac{d}{d \rho}-m_{j}^{(+)} \frac{z}{\rho}\right) R_{j}^{(+)}\right. \\
\left.+R_{i}^{(-)}\left(\rho \frac{d}{d z}-z \frac{d}{d \rho}-m_{j}^{(-)} \frac{z}{\rho}\right) R_{j}^{(-)}\right] \\
+\frac{1}{\sqrt{2}} g_{l \mu-1} C_{l \mu-1,11}^{\lambda \mu}\left[R_{i}^{(+)}\left(\rho \frac{d}{d z}-z \frac{d}{d \rho}+m_{j}^{(+)} \frac{z}{\rho}\right) R_{j}^{(+)}\right. \\
\left.\left.+R_{i}^{(-)}\left(\rho \frac{d}{d z}-z \frac{d}{d \rho}+m_{j}^{(-)} \frac{z}{\rho}\right) R_{j}^{(-)}\right]\right\} \\
\left\langle i \bar{j}\left|\hat{S}_{l \lambda \mu}\right| \mathrm{BCS}\right\rangle=2 \pi \mu{ }_{N} \sqrt{\lambda(2 \lambda+1)} g_{s} u_{i j}^{(-)} \quad(\mathrm{C} 8) \\
\int d z d \rho \rho\left\{\frac { 1 } { 2 } g _ { l \mu } C _ { l \mu } ^ { \lambda \mu } \left[10\left[R_{i}^{(+)} R_{j}^{(+)}-R_{i}^{(-)} R_{j}^{(-)}\right]\right.\right. \\
+\frac{1}{\sqrt{2}} g_{l \mu+1} C_{l \mu}^{\lambda \mu} R_{i}^{(-)} R_{j}^{(+)} \\
\left.-\frac{1}{\sqrt{2}} g_{l \mu-1} C_{l \mu-1,11}^{\lambda \mu} R_{i}^{(+)} R_{j}^{(-)}\right\} .
\end{gathered}
$$

Here $u_{i j}^{(-)}=u_{i} v_{j}-u_{j} v_{i}$ is the combination of Bogoliubov factors. The $(\rho, z)$ - dependence in the functions $R_{i}^{( \pm)}, g_{l \mu}$ and $g_{l \mu \pm 1}$ is omitted for the sake of simplicity.

For the selection rule $K_{i}+K_{j}=\mu$, the matrix elements for the transitions to the 2qp state $\alpha_{i}^{+} \alpha_{j}^{+}|\mathrm{BCS}\rangle$ read

$$
\begin{aligned}
& \left\langle i j\left|\hat{L}_{l \lambda \mu}\right| \mathrm{BCS}\right\rangle=2 \pi \mu_{N} \sqrt{\lambda(2 \lambda+1)} \frac{2 g_{l}}{\lambda+1} u_{i j}^{(-)} \\
& \text {. } \int d z d \rho \rho\left\{g_{l \mu} C_{l \mu, 10}^{\lambda \mu}\left[R_{i}^{(-)} m_{j}^{(+)} R_{j}^{(+)}-R_{i}^{(+)} m_{j}^{(-)} R_{j}^{(-)}\right]\right. \\
& +\frac{1}{\sqrt{2}} g_{l \mu+1} C_{l \mu+1,1-1}^{\lambda \mu}\left[R_{i}^{(+)}\left(\rho \frac{d}{d z}-z \frac{d}{d \rho}+m_{j}^{(-)} \frac{z}{\rho}\right) R_{j}^{(-)}\right. \\
& \left.-R_{i}^{(-)}\left(\rho \frac{d}{d z}-z \frac{d}{d \rho}+m_{j}^{(+)} \frac{z}{\rho}\right) R_{j}^{(+)}\right] \\
& +\frac{1}{\sqrt{2}} g_{l \mu-1} C_{l \mu-1,11}^{\lambda \mu}\left[R_{i}^{(+)}\left(\rho \frac{d}{d z}-z \frac{d}{d \rho}-m_{j}^{(-)} \frac{z}{\rho}\right) R_{j}^{(-)}\right. \\
& \left.\left.-R_{i}^{(-)}\left(\rho \frac{d}{d z}-z \frac{d}{d \rho}-m_{j}^{(+)} \frac{z}{\rho}\right) R_{j}^{(+)}\right]\right\}, \\
& \left\langle i j\left|\hat{S}_{l \lambda \mu}\right| \mathrm{BCS}\right\rangle=2 \pi \mu_{N} \sqrt{\lambda(2 \lambda+1)} g_{s} u_{i j}^{(-)} \\
& \int d z d \rho \rho\left\{\frac{1}{2} g_{l \mu} C_{l \mu, 10}^{\lambda \mu}\left[R_{i}^{(+)} R_{j}^{(-)}+R_{i}^{(-)} R_{j}^{(+)}\right]\right. \\
& +\frac{1}{\sqrt{2}} g_{l \mu+1} C_{l \mu+1,1-1}^{\lambda \mu} R_{i}^{(-)} R_{j}^{(-)} \\
& \left.+\frac{1}{\sqrt{2}} g_{l \mu-1} C_{l \mu-1,11}^{\lambda \mu} R_{i}^{(+)} R_{j}^{(+)}\right\} .
\end{aligned}
$$

In (C7)- C10), the functions $g_{l m}(m=\mu, \mu \pm 1)$ are

$$
g_{l m}(\rho, z)=r^{l} Y_{l m}(\theta, \phi) e^{-i m \phi} .
$$

In the case of our interest $(\lambda \mu=11)$, the transition operator has the form (3). In the above expressions, we have $l=0$ and so only the terms with $\mu-1=0$ survive. In these terms, $g_{l \mu-1}(\rho, z) \rightarrow g_{00}=1 / \sqrt{4 \pi}$ and finally we get

$$
\begin{gathered}
\left\langle i \bar{j}\left|\hat{L}_{011}\right| \mathrm{BCS}\right\rangle=\sqrt{\frac{3 \pi}{2}} \mu_{N} g_{l} u_{i j}^{(-)} \\
\int d z d \rho \rho\left[R_{i}^{(+)}\left(\rho \frac{d}{d z}-z \frac{d}{d \rho}+m_{j}^{(+)} \frac{z}{\rho}\right) R_{j}^{(+)}\right. \\
\left.+R_{i}^{(-)}\left(\rho \frac{d}{d z}-z \frac{d}{d \rho}+m_{j}^{(-)} \frac{z}{\rho}\right) R_{j}^{(-)}\right] \\
\left\langle i \bar{j}\left|\hat{S}_{011}\right| \mathrm{BCS}\right\rangle=-\sqrt{\frac{3 \pi}{2}} \mu_{N} g_{s} u_{i j}^{(-)} \int d z d \rho \rho R_{i}^{(+)} R_{j}^{(-)}, \\
\left\langle i j\left|\hat{L}_{011}\right| \mathrm{BCS}\right\rangle=\sqrt{\frac{3 \pi}{2}} \mu_{N} g_{l} u_{i j}^{(-)}(\mathrm{C} 13) \quad(\mathrm{C} 14) \\
\int d z d \rho \rho\left[R_{i}^{(+)}\left(\rho \frac{d}{d z}-z \frac{d}{d \rho}-m_{j}^{(-)} \frac{z}{\rho}\right) R_{j}^{(-)}\right. \\
\left.\left.-R_{i}^{(-)}\left(\rho \frac{d}{d z}-z \frac{d}{d \rho}-m_{j}^{(+)} \frac{z}{\rho}\right) R_{j}^{(+)}\right]\right\}
\end{gathered}
$$


$\left\langle i j\left|\hat{S}_{011}\right| \mathrm{BCS}\right\rangle=\sqrt{\frac{3 \pi}{2}} \mu_{N} g_{s} u_{i j}^{(-)} \int d z d \rho \rho R_{i}^{(+)} R_{j}^{(+)}$

$(\mathrm{C} 15)$

[1] M. N. Harakeh and A. van der Woude, Giant Resonances (Clarendon Press, Oxford, 2001).

[2] K. Heyde, P. von Neumann-Cosel, and A. Richter, Rev. Mod. Phys. 82, 2365 (2010).

[3] T. Lesinski, M. Bender, K. Bennaceur, T. Duguet, and J. Meyer, Phys. Rev. C 76, 014312 (2007).

[4] P. Vesely, J. Kvasil, V.O. Nesterenko, W. Kleinig, P.G. Reinhard, and V.Yu. Ponomarev, Phys. Rev. C 80, 031302(R) (2009).

[5] V.O. Nesterenko, J. Kvasil, P. Vesely, W. Kleinig, P.-G. Reinhard, and V.Yu. Ponomarev, J. Phys. G: Nucl. Part. Phys. 37, 064034 (2010).

[6] S. Goriely, S. Hilaire, S. Péru, M. Martini, I. Deloncle, and F. Lechaftois, Phys. Rev. C 94, 044306 (2016).

[7] V. Tselyaev, N. Lyutorovich, J. Speth, P.-G. Reinhard, and D. Smirnov, Phys. Rev. C 99, 064329 (2019).

[8] G. Kružić, T. Oishi, D. Vale and N. Paar, Phys. Rev. C 102, 044315 (2020).

[9] Li-Gang Cao, G. Colò, H. Sagawa, P.F. Bortignon, and L. Sciacchitano, Phys. Rev. C 80, 064304 (2009).

[10] N. Lo Iudice and F. Palumbo, Phys. Rev. Lett. 41, 1532 (1978).

[11] R.R. Hilton, Z.Phys. A 316, 121 (1984).

[12] D. Bohle, A. Richter, W. Steffen, A.E.L. Dieperink, N. Lo Iudice, F. Palumbo, and O. Scholten, Phys. Lett. B 137, 27 (1984).

[13] W. Ziegler, C. Rangacharyulu, A. Richter, and C. Spieler, Phys. Rev. Lett. 65, 2515 (1990).

[14] N. Lo Iudice and A. Richter, Phys. Lett. B 304, 193 (1993).

[15] N. Lo Iudice, Phys. Part. Nucl. 28, 556 (1997).

[16] N. Lo Iudice, La Rivista Del Nuovo Cimento 23 n.9, 1 (2000).

[17] A.E.L. Dieperink, Prog. Part. Nucl. Phys. 9, 121 (1983).

[18] N. Lo Iudice and Ch. Stoyanov, Phys. Rev. C 65, 064304 (2002).

[19] N. Pietralla, P. von Brentano, and A.F. Lisetskiy, Prog. Part. Nucl. Phys. 60, 225 (2008).

[20] J. Enders, P. von Neumann-Cosel, C. Rangacharyulu, and A. Richter, Phys. Rev. C 71, 014306 (2005).

[21] H. Pai, T. Beck, J. Beller, R. Beyer, M. Bhike, V. Derya, U. Gayer, J. Isaak, Krishichayan, J. Kvasil, B. Löher, V.O. Nesterenko, N. Pietralla, G. Martínez-Pinedo, L. Mertes, V.Yu. Ponomarev, P.-G. Reinhard, A. Repko, P.C. Ries, C. Romig, D. Savran, R. Schwengner, W. Tornow, V. Werner, J. Wilhelmy, A. Zilges, and M. Zweidinger, Phys. Rev. C 93, 014318 (2016).

[22] M. Guttormsen, L.A. Bernstein, A. Bürger, A. Görgen, F. Gunsing, T.W. Hagen, A. C. Larsen, T. Renstrøm, S. Siem, M. Wiedeking, and J.N. Wilson, Phys. Rev. Lett. 109, 162503 (2012).

[23] E.B. Balbutsev, I.V. Molodtsova, and P. Schuck, Nucl. Phys. A 872, 42 (2011).

[24] E.B. Balbutsev, I.V. Molodtsova, and P. Schuck, Phys. Rev. C 91, 064312 (2015).

[25] E.B. Balbutsev, I.V. Molodtsova, and P. Schuck, Phys.
Rev. C 97, 044316 (2018).

[26] I.V. Molodtsova and E.B. Balbutsev, Eur. Phys. J. Web of Conf. 194, 04004 (2018).

[27] E.B. Balbutsev, I.V. Molodtsova, and P. Schuck, Eur. Phys. J. Web of Conf. 194, 04005 (2018).

[28] E.B. Balbutsev, I.V. Molodtsova, A.V. Sushkov, N.Yu. Shirikova, and P. Schuck, arXiv:1902.05275 [nucl-th], 2019.

[29] E.B. Balbutsev, I.V. Molodtsova, and P. Schuck, Phys. Atom. Nucl. 83, 212 (2020).

[30] C. Wesselborg, P. von Brentano, K.O. Zell, R.D. Heil, H.H. Pitz, U.E.P. Berg, U. Kneissl, S. Lindenstruth, U. Seemann, and R. Stock, Phys. Lett. B 207, 22 (1988).

[31] J. Margraf, T. Eckert, M. Rittner, I. Bauske, O. Beck, U. Kneissl, H. Maser, H. H. Pitz, A. Schiller, P. von Brentano, R. Fischer, R.-D. Herzberg, N. Pietralla, A. Zilges, and H. Friedrichs, Phys. Rev. C 52, 2429 (1995).

[32] S. Valenta, B. Baramsai, T.A. Bredeweg, A. Couture, A. Chyzh, M. Jandel, J. Kroll, M. Krtička, G. E. Mitchell, J.M. O'Donnell, G. Rusev, J.L. Ullmann, and C. L. Walker, Phys. Rev. C 96, 054315 (2017).

[33] T. Renstrøm, H. Utsunomiya, H.T. Nyhus, A.C. Larsen, M. Guttormsen, G.M. Tveten, D.M. Filipescu, I. Gheorghe, S. Goriely, S. Hilaire, Y.-W. Lui, J.E. Midtbø, S. Péru, T. Shima, S. Siem, and O. Tesileanu, Phys. Rev. C 98, 054310 (2018).

[34] A.S. Adekola, C.T. Angell, S.L. Hammond, A. Hill, C.R. Howell, H.J. Karwowski, J.H. Kelley, and E. Kwan, Phys. Rev. C 83, 034615 (2011).

[35] P. Ring and P. Schuck, The Nuclear Many-Body Problem (Springer-Verlag, New York, Heidelberg, Berlin, 1980).

[36] N. Van Giai and H. Sagawa, Phys. Lett. B 106, 379 (1981).

[37] V.G. Soloviev, Theory of Atomic Nuclei (Pergamon Press, Oxford, 1976).

[38] B.R. Mottelson and S.G. Nilsson, Mat. Fys. Skr. Dan. Vid. Selsk., 1, No. 8 (1959).

[39] M. Bender, P.-H. Heenen, and P.-G. Reinhard, Rev. Mod. Phys. 75, 121 (2003).

[40] J. R. Stone and P.-G. Reinhard, Prog. Part. Nucl. Phys. 58, 587 (2007).

[41] A. Repko, J. Kvasil, V.O. Nesterenko, and P.-G. Reinhard, arXiv:1510.01248 (nucl-th), 2015.

[42] A. Repko, J. Kvasil, V.O. Nesterenko, and P.-G. Reinhard, Eur. Phys. J. A 53, 221 (2017).

[43] A. Repko, J. Kvasil, and V.O. Nesterenko, Phys. Rev. C 99, 044307 (2019).

[44] J. Kvasil, A. Repko, and V.O. Nesterenko, Eur. Phys. J. A 55, 213 (2019).

[45] J. Bartel, P. Quentin, M. Brack, C. Guet, and H.-B. Håkansson, Nucl. Phys. A 386, 79 (1982).

[46] P. Klüpfel, P.-G. Reinhard, T.J. Bürvenich, and J.A. Maruhn, Phys. Rev. C 79, 034310 (2009).

[47] P. Sarriguren, E. Moya de Guerra, and R. Nojarov, Phys. Rev. C 54, 690 (1996).

[48] P.-G. Reinhard, B. Schuetrumpf, and J.A. Maruhn, Com- 
put. Phys. Commun. 258, 107603 (2021).

[49] Database http://www.nndc.bnl.gov/nudat2/chartNuc.jsp

[50] P.-G. Reinhard, private communication.

[51] M. Bender, K. Rutz, P.-G. Reinhard, and J.A. Maruhn, Eur. Phys. J. A 8, 59 (2000).

[52] N. Pietralla, P. von Brentano, R.-D. Herzberg, U. Kneissl, J. Margraf, H. Maser, H. H. Pitz, and A. Zilges, Phys. Rev. C 52, R2317 (1995).

[53] V.G. Soloviev, A.V. Sushkov, N.Yu. Shirikova, and N. Lo Iudice, Nucl. Phys. A 600, 155 (1996).

[54] D. Frekers, H.J. Wörtche, A. Richter, R. Abegg, R.E. Azuma, A. Celler, C. Chan, T.E. Drake, R. Helmer, K.P. Jackson, J.D. King, C.A. Miller, R. Schubank, M.C. Vetterli, and S. Yen, Phys. Lett. B 244, 178 (1990).

[55] H.L. Wörtche, Ph.D. thesis, Technischen Hochschule
Darmstadt, Germany, 1994.

[56] W. Kleinig, V.O. Nesterenko, J. Kvasil, P.-G. Reinhard and P. Vesely, Phys. Rev. C78, 044313 (2008).

[57] A. Repko, P.-G. Reinhard, V. O. Nesterenko, and J. Kvasil, Phys. Rev. C 87, 024305 (2013).

[58] A. Repko, V.O. Nesterenko, J. Kvasil, and P.-G. Reinhard, Eur. Phys. J. A 55, 242 (2019).

[59] M. Goldhaber and E. Teller, Phys. Rev. 74, 1046 (1948).

[60] H. Steinwedel and J. H. D. Jensen, Z. Naturforsch. 5a, 413 (1950).

[61] S. F. Semenko, Sov. J. Nucl. Phys. 34, 356 (1981).

[62] A. Bohr and B. R. Mottelson, Nuclear Structure, Vol. 1 (Benjamin, New York, 1969). 